\title{
Thermodynamics of pairing in mesoscopic systems
}

\author{
Tony Sumaryada and Alexander Volya \\ Department of Physics, Florida State University, Tallahassee, FL 32306-4350, USA
}

(Dated: October 24, 2018)

\begin{abstract}
Using numerical and analytical methods implemented for different models we conduct a systematic study of thermodynamic properties of pairing correlation in mesoscopic nuclear systems. Various quantities are calculated and analyzed using the exact solution of pairing. An in-depth comparison of canonical, grand canonical, and microcanonical ensemble is conducted. The nature of the pairing phase transition in a small system is of a particular interest. We discuss the onset of discontinuity in the thermodynamic variables, fluctuations, and evolution of zeros of the canonical and grand canonical partition functions in the complex plane. The behavior of the Invariant Correlational Entropy is also studied in the transitional region of interest. The change in the character of the phase transition due to the presence of magnetic field is discussed along with studies of superconducting thermodynamics.
\end{abstract}

PACS numbers: 21.60.Cs, 24.10.Cn, 71.10.Li

\section{INTRODUCTION}

Pairing correlations and related superconducting or superfluid properties are robust features of quantum manybody systems. In physics anywhere from quarks to stars it is hard to find systems that under certain conditions do not exhibit pairing correlations. The Cooper phenomenon [1], namely the instability against formation of particle-pairs in a macroscopic Fermi-system under an arbitrarily weak attractive force, is a primary reason for thriving of pairing.

Pairing in mesoscopic systems, such as atomic nuclei [2], metal clusters [3, 4, 5], ultra small grains [6], quantum dots [7], interacting spins [8, 9], has attracted a lot of attention recently. Indeed, questions of phase transitions 11, 10, 11, 12, 13], interplay with other collective modes [14, 15], continuum effects [16] and thermodynamical properties of small systems are important for the present day science and technology.

In this work we conduct a systematic study of thermodynamics of pairing correlations in small systems. We use two-types of model Hamiltonians of lower and higher symmetry where the pairing problem is solved exactly and all quantum states are identified. We use a quasispin algebra with the effective numerical implementation to obtain a full solution for systems ranging in size from a few particles to as large as over a hundred of particles. The traditional BCS solution is also considered for comparison. Using these results we compare different thermodynamic ensembles: microcanonical, canonical and grand canonical. The differences indicate a mesoscopic nature of the system [17, 18, 19] and diminish in the macroscopic limit. Some discrepancies observed in thermodynamics are related to non-thermal nature of the pure pairing interaction [20] and raise questions of equilibration and thermalization. Through thermodynamic ensembles and using invariant correlational entropy we study and analyze the pairing phase transition as a function of temperature or excitation energy, magnetic field, size of the system, and pairing strength.
We further explore the evolution of zeros in the complex temperature plane for the canonical ensemble [21, 22, 23, 24, where recent findings established clear correlations of pair breaking with peaks in entropy and branches of complex temperature roots approaching real axis [2, 18, 25]. We extend this discussion with consideration of the phase transition based on the Yang-Lee theory [26, 27, 28]. The study of the system in the magnetic field, evolution of zeros in the partition function as a function of the field strength, spin fluctuations and the change of the phase transition type are particularly interesting.

The presentation below is structured as follows: we first introduce the pairing Hamiltonian, identify properties of the pairing problem and define models for our study in Sec. III. In Sec. III we consider a BCS approximation which shows the generic features of a paired system. The bulk of the work is presented in Sec. IV] and its subsections, where different methods are introduced, discussed and compared. In Sec. VI we concentrate on the effects that external magnetic field or rotation have on the properties of paired systems; this includes the classification of the phase transitions using the distribution of zeros in the partition functions.

\section{PAIRING HAMILTONIAN}

We approach the pairing problem by defining a pair of two single-particle states denoted here as 1 and $\tilde{1}$. This pair-wise identification can be based on an arbitrary symmetry; however, the fundamental symmetry with respect to time reversal is the most common. For this work we assume a pair as two particles in time-conjugated singleparticle states that due to this symmetry have identical energies. Using the language of the second quantization the pair creation and annihilation operators are $p_{1}^{\dagger}=a_{1}^{\dagger} a_{\tilde{1}}^{\dagger}$ and $p_{1}=a_{\tilde{1}} a_{1}$, respectively. Here the $a_{1}^{\dagger}$ and $a_{1}$ are single-particle creation and annihilation operators with the usual fermion commutation rules. The pair is 
labeled by the same single-particle index 1 , and is invariant under the time conjugation, $p_{1}=p_{\tilde{1}}$, since $a_{\tilde{1}}=a$.

The algebra of the pair operators on a pair-state 1 (a pair of orbitals 1 and $\tilde{1}$ ) is identical to that of an $\mathrm{SU}(2)$ spin algebra called quasi-spin, in general the commutation relations are

$$
\left[p_{1}^{\dagger}, p_{2}\right]=2 \delta_{12} p_{1}^{z},
$$

where

$$
p_{1}^{z}=\left(n_{1}-\frac{1}{2}\right),
$$

the operator related to the particle number $n_{1}=\left(a_{1}^{\dagger} a_{1}+\right.$ $\left.a_{\tilde{1}}^{\dagger} a_{\tilde{1}}\right) / 2$ operator for the pair-state 1.

A pair-state $(1, \tilde{1})$ occupied by a pair or completely empty correspond to quasi-spin $1 / 2$ with projections $p_{1}^{z}=1 / 2$ and $p_{1}^{z}=-1 / 2$, respectively. Alternatively, these states are referred to as states with seniority $s_{1}=0$ identifying the number of unpaired nucleons in the pairstate 1 . The states with one unpaired particle correspond to $s_{1}=1$ and to zero quasi-spin.

The most general form of the two-body Hamiltonian that describes motion of pairs at fixed particle number is

$$
H=2 \sum_{1>0} \epsilon_{1} n_{1}-\sum_{1,2>0} G_{12} p_{1}^{\dagger} p_{2},
$$

where the summation runs over pair-orbitals, denoted as $1>0, \epsilon_{1}$ are single-particle energies, and $G_{12}=G_{21}$ determines the strength of pair scattering. Using the quasi-spin the same Hamiltonian can be written as

$H=\sum_{1>0} \epsilon_{1}+\sum_{1>0} 2\left(\epsilon_{1}-\frac{G_{11}}{2}\right) p_{1}^{z}-\sum_{12>0} G_{12}\left(\vec{p}_{1} \cdot \vec{p}_{2}-p_{1}^{z} p_{2}^{z}\right)$

The problem is analogous to the Heisenberg model of $\Omega / 2-s$ interacting spins $|\vec{p}|=1 / 2$, with the Zeeman splitting created by the single-particle energies. The $\Omega / 2$ stands here for the total number of double-degenerate levels and $s=\sum_{1} s_{1}$ represents the total seniority. Because of the magnetic-field like splitting the total quasispin vector $\vec{p}=\sum_{1>0} \vec{p}_{1}$ is not conserved, while the remaining cylindrical symmetry allows for the conservation of the $z$-projection $p^{z}=N / 2-\Omega / 4$, equivalent to the total particle number $N=2 \sum_{1>0} n_{1}$.

The eigenstates of the Hamiltonian (3) are identified by the set of $\Omega / 2$ seniorities $\mathbf{S}=\left\{s_{1}\right\}$ denoting the available and blocked pair-states. In the language of the spin model (4) seniorities represent the number of spin $1 / 2$ particles in the system, thus totally removing all blocked states from interaction. The Hamiltonian within a certain seniority partition $\mathbf{s}$ is given as

$$
\begin{array}{r}
H_{\mathbf{s}}=\sum_{1>0} s_{1} \epsilon_{1}+2 \sum_{1>0}^{\mathbf{s}} n_{1}\left(\epsilon_{1}-\frac{G_{11}}{2}\right) \\
-\sum_{1 \neq 2}^{\mathbf{s}} G_{12}\left(\vec{p}_{1} \cdot \vec{p}_{2}-p_{1}^{z} p_{2}^{z}\right)
\end{array}
$$

where the upper summation limit $\mathbf{s}$ implies that all blocked states with $s_{1}=1$ are excluded.

Since each unpaired particle doubles the degeneracy of the many-body state the total degeneracy of a given eigenstate is $g_{\mathbf{s}}=2^{s}$. With other symmetries, beyond the time reversal, the degeneracy of states can be higher. Additional degeneracies such as the one due to the rotational symmetry can further reduce the problem to larger values of the quasi-spin. In the spherical shell model within a given $j$-shell there are total of $\omega_{j}=j+1 / 2$ time-conjugate pair states, and the total quasi-spin is preserved by the pairing interaction. For such $j$-shell a quasi-spin vector $\vec{p}_{j}=\frac{1}{2} \sum_{m} \vec{p}_{j m}$ can be introduced which together with the number operator for this level and its own hermitian conjugate again forms an $\mathrm{SU}(2)$ group. The independence of matrix elements and quasispin operators on magnetic sub-states allows to rewrite the Hamiltonian (3) as

$$
H=\sum_{j} \epsilon_{j} N_{j}-\sum_{j j^{\prime}} V_{j j^{\prime}} P_{j}^{\dagger} P_{j^{\prime}},
$$

where for the reasons of the two-particle state normalization a pair operator and interaction matrix elements are redefined as follows

$$
\begin{gathered}
\vec{P}_{j}=\frac{1}{2 \sqrt{\omega_{j}}} \sum_{m} \vec{p}_{j m}, \\
V_{j j^{\prime}}=\sqrt{\omega_{j} \omega_{j^{\prime}}} G_{j j^{\prime}} .
\end{gathered}
$$

The exact diagonalization of the pairing Hamiltonian (3) or (6), depending on the symmetries of the model, is performed using the quasi-spin algebra. The ability to obtain all many-body states with a relatively simple exact treatment of pairing is an important component in this study. The more detailed discussion of the seniority based diagonalization can be found in Ref. [29, 30, 31]. We refer to the exact treatment of pairing as EP. The applications of algebraic methods extend far beyond our models; treatments of proton-neutron pairing as well as more exotic forms of pairing-type Hamiltonians are discussed in Ref. [32, 33, 34, 35, 36, 37, 38, 39, 40]. Other methods of exact solution, analogies with boson-fermion models and electrostatic analogies should be mentioned [41, 42, 43, 44, 45, 46] .

Below in Sec. (IV) we introduce thermodynamic ensembles and discuss thermodynamic variables used to study the many-body system that undergoes pairing phase transition. For each of the cases we construct the partition function exactly based on the full numerical solution to the pairing problem. As our examples we consider two basic types of systems. The picket-fence (or ladder system) which has $\Omega / 2$ equally spaced doubledegenerate levels, where the total fermion capacity is $\Omega$. The level spacing is chosen as the unit of energy. The picket-fence model is a minimal symmetry system with the time reversal only; therefore the degeneracy of each 
eigenstate $\alpha$ is $g_{\alpha \mathrm{s}}=2^{s}$. A second model with only two levels, but of large degeneracy, represents an opposite "high symmetry" case. Spacing between the two levels is again taken as the unit of energy. Due to additional symmetry, the degeneracy of many-body states is higher. The action of the pairing Hamiltonian is limited to either diagonal or level to level pair transfer. For the two-level system with an appropriate selection of the basis states the Hamiltonian matrix is tri-diagonal. This facilitates substantially the numerical treatment, making determination of all many-body states in systems with a hundred or more particles possible. The two types of model spaces with total occupancy $\Omega$, the particle number $N$, and the constant pairing strength $G$ constitute the set of input parameters in this study. Introduction of the magnetic field in SecVI does not require a separate diagonalization, however requires determination of the total spin projections onto an axis parallel to the direction of the field. We note that the total number of many-body states is $\boldsymbol{\Omega}=\frac{\Omega !}{N !(\Omega-N) !}$.

\section{BCS}

The BCS approximation is the common approach to tackle the pairing problem. While this method is asymptotically exact in thermodynamic limit it still produces remarkably good results for smaller systems. The BCS method assumes the presence of a condensate and approximates the dynamics of interacting particles (3) with a motion of independent quasi-particles. Although most of the issues that we intend to address in this work can not be fully explored within the BCS picture due to its limitations, the method is a good benchmark for many of the questions and an excellent guidance to the dynamical regions of interest. Below we review the approach while stressing some of the key elements relevant to this work.

Within the BCS theory the general pairing Hamiltonian in Eq. (3) is brought to an approximate single particle form using Bogoliubov's transformation. The parameters of the transformation, the set of gaps $\Delta_{1}$ and chemical potential $\mu$, are determined via gap equations

$$
\Delta_{1}=\frac{1}{2} \sum_{2>0} G_{12} \frac{\Delta_{2}}{e_{2}}
$$

and the chemical potential is given by the particle number

$$
N=2 \sum_{1>0} n_{1} \quad \text { where } \quad n_{1}=\frac{1}{2}\left(1-\frac{\varepsilon_{1}}{e_{1}}\right) .
$$

For simplicity of notations we introduce single particle energies shifted by the chemical potential and the diagonal interaction strength $\varepsilon_{1}=\epsilon_{1}-\mu-G_{11} / 2$. The result of the Bogoliubov transformation is the spectrum of states given by the independent quasi-particle excitations with energies

$$
e_{1}=\sqrt{\varepsilon_{1}^{2}+\Delta_{1}^{2}}
$$

The total energy of the paired system is

$$
E=2 \sum_{1>0}\left(\epsilon_{1}-\frac{G_{11}}{2}\right) n_{1}-\sum_{1,2>0} G_{12} \frac{\Delta_{1} \Delta_{2}}{4 e_{1} e_{2}} .
$$

As earlier, the summations here go over the pair-states.

In this work for all our models we use a constant pairing strength $G_{11} \equiv G$ which due to Eq. (18) leads to a constant pairing gap for all single particle pairs, $\Delta_{1} \equiv \Delta$. A single parameter for the interaction strength, in our view, allows for the most transparent study of the important features, the results are generic, and the methods of BCS and EP are applicable to general situations. For constant pairing the BCS gap equation and the energy are a textbook examples:

$$
1=\frac{G}{2} \sum_{1>0} \frac{1}{e_{1}}, \quad E=2 \sum_{1>0}\left(\epsilon_{1}-\frac{G}{2}\right) n_{1}-\frac{\Delta^{2}}{G} .
$$

To accommodate the cases with higher symmetry following Eq. (17) it is convenient to introduce $V=\omega G$ where $\omega$ is the pair degeneracy which is level independent in both picket-fence $\left(\omega_{j}=1\right)$ and two-level $\left(\omega_{j_{1}}=\omega_{j_{2}} \equiv \omega\right)$ models.

The particle number non-conservation intrinsic to the Bogoliubov transformation is one of the problems associated with the BCS applications to mesoscopic systems. Furthermore, in a system with discrete levels Eq. (8) may not have a solution, with the exception of a trivial case $\Delta_{1}=0$. Formally, this transitional point [47] corresponds to the critical interaction strength where the largest eigenvalue of the matrix built from the elements $\left(G_{12} \varepsilon_{1}+G_{21} \varepsilon_{2}\right) /\left(4 \varepsilon_{1} \varepsilon_{2}\right)$ is equal to unity. The interpretation of this is that at a low pairing strength the pairing is too weak to overcome gaps in the single particle spectrum which leads to a normal state. This situation is again specific to small systems where it appears in contrast to the Cooper instability [1]. The total absence of the pairing correlations below the critical pairing strength is a second major drawback of the BCS approach in mesoscopic systems. Exact solutions indicate a gradual dissipation of pairing correlations extending almost to zero strength [2, 47, 48, 49]. The critical pairing strength as determined by the BCS is still an important parameter identifying the location of the mesoscopic phase transition.

An analytic solution to the BCS equations can be obtained for the system of two levels defined above. For a half-occupied system the chemical potential due to the particle-hole symmetry is an exact average of the monopole-renormalized single-particle energies $\mu=$ $\left(\epsilon_{1}+\epsilon_{2}-G\right) / 2$. Thus,

$$
\Delta^{2}=V^{2}-\left(\frac{\Delta \epsilon}{2}\right)^{2}, \quad \Delta \epsilon=\epsilon_{1}-\epsilon_{2} .
$$

The introduction of the renormalized strength $V$ makes this equation independent of $\Omega$.

In Fig. 1(a) the BCS gap is plotted as a function of energy for a two-level model following Eq. (12). The 
curve has a square-root discontinuity at the critical pairing strength $V_{c r}=0.50$ in the units of level spacing. The concept of the gap does not appear in the exact solution, however this quantity can be deduced from the energy associated with paring correlations. The second curve in the same figure shows the gap computed through Eq. (11) where energy and occupation numbers are obtained from the exact solution. The difference between these two curves depicts the shortcoming of the BCS when applied to a small system; for related discussions and comparison of BCS with exact techniques see Refs. [50, 51, 52]. In Fig. 1(b) an alternative view on the EP-BCS comparison is given. Here we show the energy difference per particle between BCS and EP as a function of the pairing strength for $\mathrm{N}=20$ and 100 particles. As the particle number grows the BCS and EP become equivalent. The peak in the BCS-EP discrepancy appears in the pairing phase transition region, around $V_{c r} \approx 0.6$ which is close to an analytically obtained BCS value of 0.5 . The discrepancy in $V_{c r}$ is known to arise from the pair-vibrations and other renormalizations of the BCS ground state [53, 54].

For our second (picket-fence) model, the critical pairing strength can be determined in the case of a halfoccupied system with an even number of levels through the sum of a harmonic series

$$
G_{c r}=\frac{\Delta \epsilon}{\left(\sum_{n=1}^{(\Omega / 2+1) / 2} \frac{1}{n}+\ln 4\right)},
$$

which in the limit of a large number of levels converges to zero logarithmically $G_{c r} \sim \Delta \epsilon / \ln \Omega$. We remind here that formally for this model $G=V$ since $\omega_{j}=1$ and $\Omega / 2$ equals to the number of levels. This logarithmic dependence in the macroscopic limit is related to the exponential dependence of the gap on the pairing strength and density of states near the Fermi surface, which represents the Cooper instability.

We conclude this section with a note on the BCS approach at finite temperature $T=1 / \beta$. By modeling the thermodynamics of quasiparticles with non-interacting Fermi gas we obtain a modified version of the Eq. (11)

$$
1=\frac{G}{2} \sum_{1>0} \frac{\tanh \left(\frac{\beta}{2} e_{1}\right)}{e_{1}},
$$

where quasiparticle energies are of the form (10). A related discussion of thermodynamic treatment within the grand canonical partition function and following from it thermal BCS is presented in Sec. IVB

For the two-level, half occupied model the temperature dependence of the critical pairing strength is given by

$$
V_{c r}=\frac{\Delta \epsilon}{2} \operatorname{coth}\left(\frac{\beta \Delta \epsilon}{4}\right)
$$
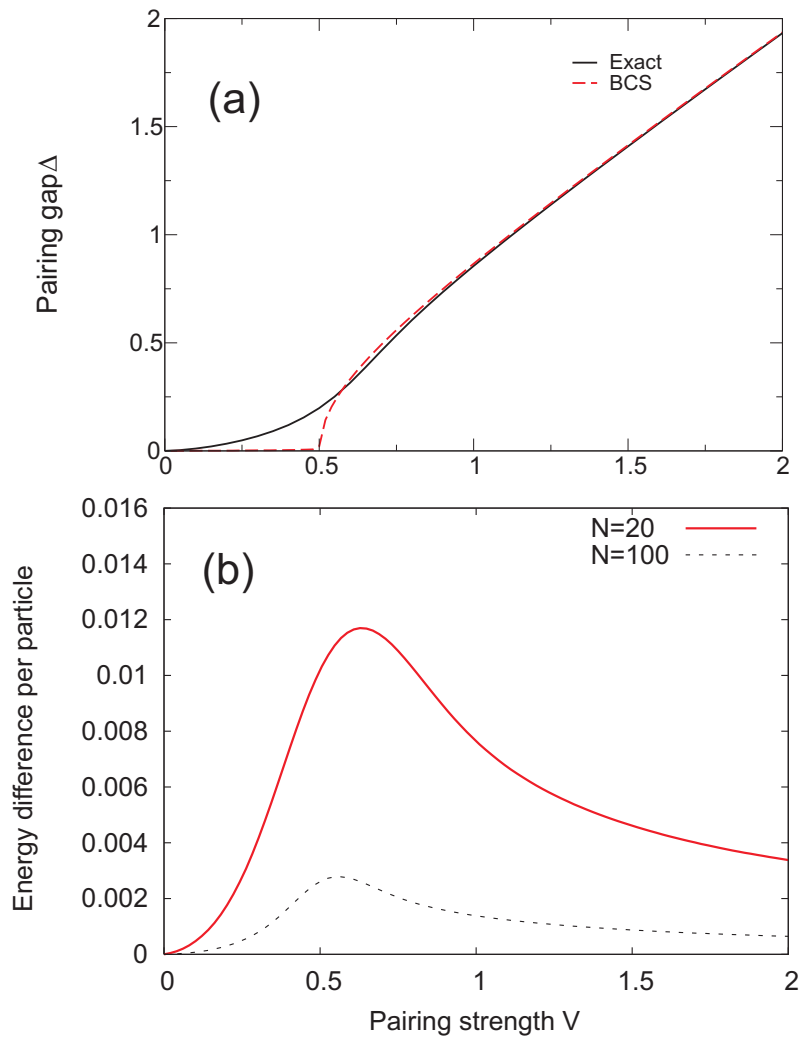

Figure 1: (Color online) In the upper panel the BCS pairing gap is shown as a function of the pairing strength for the twolevel, half-occupied system with 20 particles. In the lower panel the energy difference per particle between BCS and the exact result is shown as a function of the pairing strength for the same $N=20$ system and is compared with the results for a larger half-occupied two-level model containing 100 particles.

\section{STATISTICAL TREATMENT}

Statistical properties of a many-body systems are addressed using normalized density operators [55], usually referred to as statistical operators $\hat{w}[56]$ and defined as

$$
\hat{w}(E, N)=\frac{1}{Z} \delta(E-\hat{H}) \delta(N-\hat{N})
$$

for the microcanonical,

$$
\hat{w}(\beta, N)=\frac{1}{\mathcal{Z}} \exp (-\beta \hat{H}) \delta(N-\hat{N})
$$

for the canonical, and

$$
\hat{w}(\beta, \mu)=\frac{1}{\mathbf{Z}} \exp (-\beta(\hat{H}-\mu \hat{N}))
$$

for the grand canonical ensemble. In the above definitions the parameter $\beta=1 / T$ refers to an inverse temperature and $\mu$ corresponds to the chemical potential. Here we use units where the Boltzmann constant is equal to unity, allowing units of energy to be used for temperature. The normalization constants $Z, \mathcal{Z}$, and $\mathbf{Z}$ are the 
partition functions for the corresponding ensembles; so that the statistical operators are normalized by the trace $\operatorname{Tr}(\hat{w})=1$. The statistical averages are calculated as

$$
\langle\hat{O}\rangle=\operatorname{Tr}(\hat{O} \hat{w})
$$

The entropy for the above ensembles is defined as

$$
S=-\langle\ln (\hat{w})\rangle=-\operatorname{Tr}(\hat{w} \ln \hat{w}) .
$$

The above definition is strictly speaking applicable only for thermally equilibrated system which makes thermodynamical Boltzmann-Gibbs entropy discussed below equivalent to the von-Neumann entropy of a quantum ensemble in Eq. (20). A new light on complexity of quantum states in non-thermalized or non-equilibrated systems can be obtained with the invariant correlational entropy [57] (ICE) that also appears to be a good tool to study the phase transitions in mesoscopic systems [58, 59]. The correlational entropy is defined through the behavior of the microcanonical density matrix (16) for each individual quantum state in response to a noise in an external parameter. For the purposes of this work we consider pairing strength $V$ to be this external parameter. The variations in $V$ within the interval $[V, V+\delta V]$ result in an averaged density operator

$$
\hat{w}_{\alpha}=\frac{1}{\delta V} \int_{V}^{V+\delta V} \hat{w}_{\alpha}(V)
$$

where the weight operator $\hat{w}_{\alpha}$ is a density operator for an individual quantum state $\alpha$ followed with evolution of $V$, for a fixed parameter $V$ this is a projection operator. The averaged statistical weight matrix is used to obtain the ICE via Eq. (20).

The quality or applicability of a given thermodynamic approach to a small system is often under question. While in some studies various ensembles are used interchangeably, there are significant dangers on this path. Our investigations below not only show up the pairing phase transition and its evolution as a function of the particle number but also draw attention to some subtle differences in thermodynamic treatments.

\section{A. Canonical ensemble}

Given an exact solution to the pairing problem via diagonalization in the seniority scheme, Sec. II the formal definition (17) can be written explicitly for the eigenstates labeled by $\alpha$ and $\mathbf{s}$ :

$$
\begin{gathered}
w_{\alpha \mathbf{s}}=\frac{1}{\mathcal{Z}} \exp \left(-\beta E_{\alpha \mathbf{s}}\right), \quad \text { where } \\
\mathcal{Z}(\beta, N)=\sum_{\alpha \mathbf{s}} g_{\alpha \mathbf{s}} \exp \left(-\beta E_{\alpha, \mathbf{s}}\right)
\end{gathered}
$$

is the canonical partition function. The ensemble average (19) for any quantity is given as

$$
\langle O\rangle=\sum_{\alpha \mathbf{s}} g_{\alpha s} w_{\alpha s}\langle\alpha \mathbf{s}|O| \alpha \mathbf{s}\rangle,
$$

where $\langle\alpha \mathbf{s}|O| \alpha \mathbf{s}\rangle$ is the quantum-mechanical expectation value for the corresponding operator in the eigenstate $\alpha$ with the seniority set $\mathbf{s}$. The entropy is given via the usual expression

$$
S=-\sum_{\alpha \mathbf{s}} g_{\alpha s} w_{\alpha s} \ln \left(w_{\alpha s}\right)
$$

The reader may be familiar with the following set of traditional thermodynamic relations [60]

$$
\langle E\rangle=-\frac{\partial}{\partial \beta} \ln (\mathcal{Z}),
$$

the entropy $S$ can be found directly from the statistical definition (20)

$$
S=\ln \mathcal{Z}+\beta\langle E\rangle=-\frac{\partial F}{\partial T} .
$$

The Helmholtz free energy is defined as

$$
F=-T \ln (\mathcal{Z})=\langle E\rangle-T S .
$$

The Eq. (25) involves a derivative, however in our calculations we avoid numerical differentiations always going back to the definition (23). For example specific heat is computed using its relation to the energy fluctuations $\left\langle(E-\langle E\rangle)^{2}\right\rangle$,

$$
C=\left(\frac{\partial\langle E\rangle}{\partial T}\right)=\beta^{2} \frac{\partial^{2} \ln \mathcal{Z}}{\partial \beta^{2}}=\beta^{2}\left\langle(E-\langle E\rangle)^{2}\right\rangle .
$$

The results of our study based on the canonical ensemble are shown in Fig. 2,8. In Fig. 2 (a - d) free energy, entropy, energy, and energy fluctuation of the ladder system with 12 levels and 12 particles are shown as a function of temperature, similar study may be found in [2, 61 and references therein. The critical pairing strength for this model from BCS, Eq. (13), at zero temperature is $V_{c r}=0.27$. The curves correspond to different pairing strengths showing various conditions: weak pairing with about half the critical pairing strength $V=0.13$; pairing strength above the critical value $V=0.6$; and strong pairing $V=1$. All of the plots show essentially similar trends: there is a sharp change in each of the quantities as a function of temperature in a certain region. This region is associated with the phase transition from the paired to the normal state. Most transparently it can be seen in 2(e) where it is associated with the peak in heat capacity. The critical temperature $T_{c r}$ depends on the pairing strength. It can be observed that the transitional region for strong pairing $\left(V>V_{c r}\right.$ for $\left.T=0\right)$ is roughly consistent with the $\mathrm{BCS}$, which gives $T_{c r}=2.7$ and 1.3 for $V=1$ and 0.6 , respectively. Naturally, the 
stronger pairing interactions support the superconducting state at higher temperature or excitation energy. For weak pairing the transitional behavior is present at zero temperature. This is consistent with the earlier finding that pairing correlations appear in the ground state even for small $V$. The decline of weak pairing $(V \leq 0.13)$ phase is still associated with the peak in heat capacity which becomes smaller as the pairing strength is weakened, while staying essentially at the same $T_{c r} \sim 1.3$.
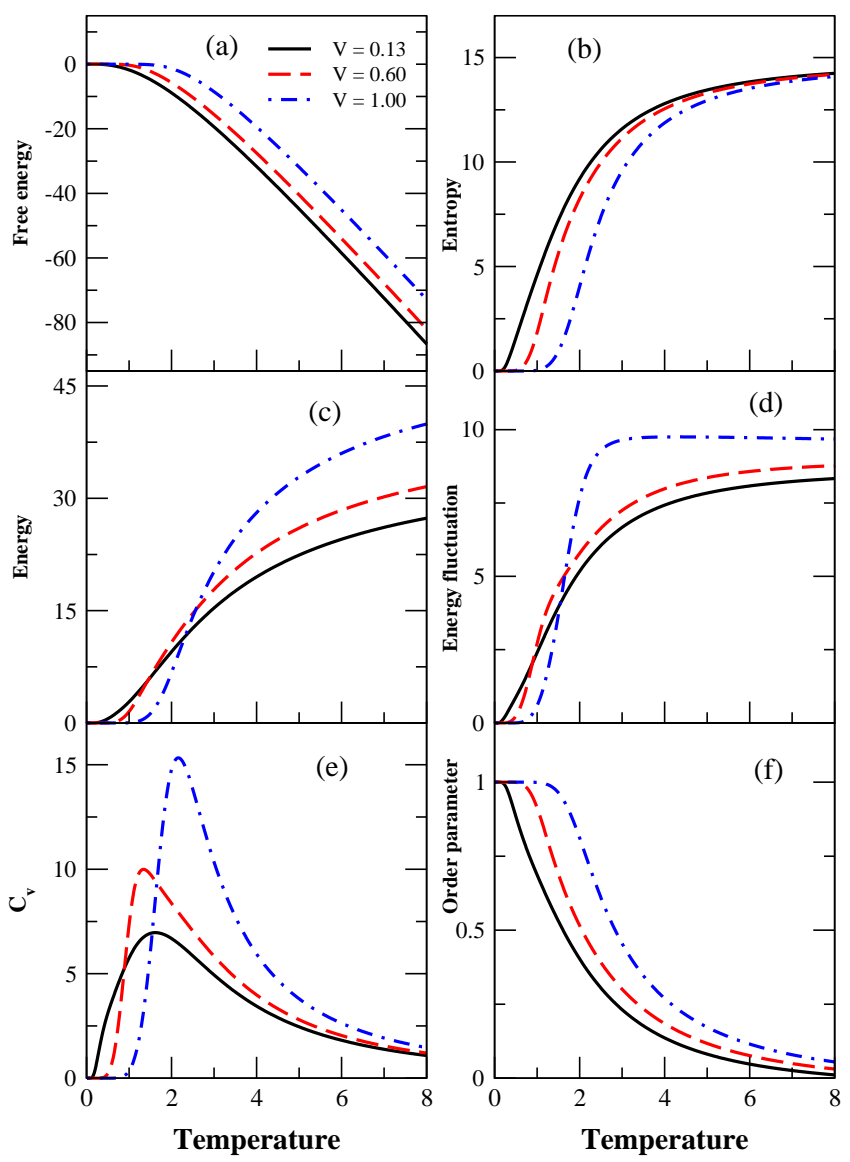

Figure 2: (Color online) (a) Free energy, (b)Entropy, (c)Energy, (d) Energy fluctuations, (e) Specific heat, and (f) Order parameter of a ladder system with 12 levels and 12 particles as a function of temperature.

The phase diagram can be further explored by considering an order parameter which we define here as a fraction of paired particles $\psi=(N-\langle s\rangle) / N$, the $\langle s\rangle$ is the ensemble-averaged value of the total seniority. The dependence of the order parameter on temperature, shown in Fig. 2(f), shows that the fraction of superconducting pairs drops sharply in the transitional region which is also identified by the critical behavior of other thermodynamic quantities.

The contour plot of the order parameter as a function of the pairing strength and temperature is shown in Fig. 3. The shaded area in the upper left corresponds to the high percentage of particles in the condensate, which occurs at low temperature and high pairing strength; while in the opposite limit the superconducting state disappears. The solid line indicates the phase boundary as follows from the BCS approximation. We note that at zero temperature the fraction of superconducting particles is high even at zero pairing strength this special point corresponds to the absence of two-body interactions which results in pair-wise Fermi occupation of time-reversed orbitals.

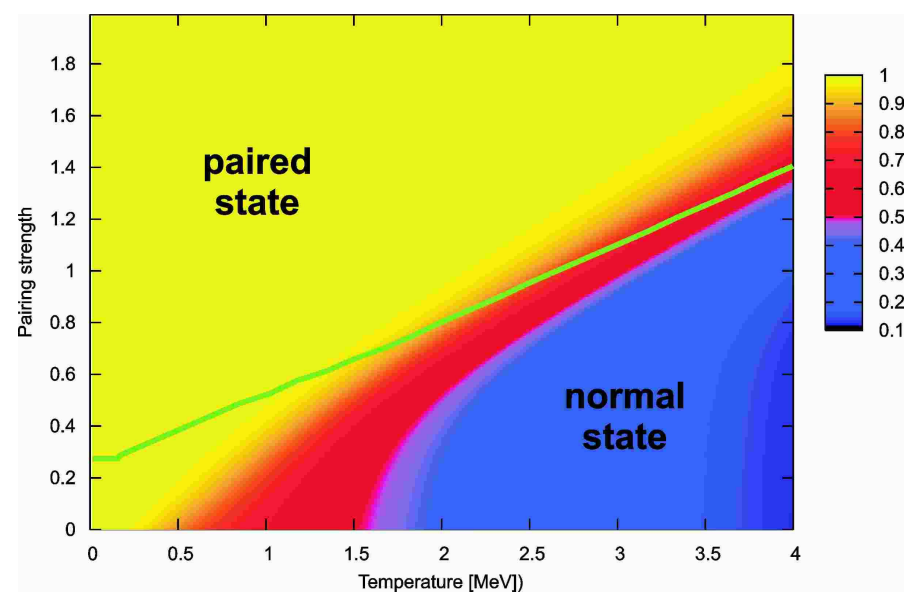

Figure 3: (Color online) The contour plot of the order parameter as a function of the pairing strength and temperature. Half occupied 12-level system is shown. The line separates normal and paired regions based on the BCS equation.

Throughout this work we mainly discuss systems with an even particle number; we found that the difference between odd and even systems in the critical region of interest is small. Most of the distinction occurs at zero temperature where degeneracy of an odd-particle ground state and non-zero spin are important. This can be seeing in Fig. 4 where we compare the entropy and specific heat as a function of temperature for $N=11$ and $N=1212$ level ladder systems.

The transition to the thermodynamic limit is explored for a two-level system in Fig. 5. Unless noted otherwise, in our study we select exactly half-occupied systems with $N=\Omega / 2$. The region of interest is identified by the peak in heat capacity seen in Fig. 5(b). With the increased particle number this peak becomes sharper as expected in the macroscopic limit, where the phase transition is represented by a discontinuity. Another interesting remark can be made about the location of the peak. Following Eq. (15) within the BCS approximation the location of the phase transition for a half-occupied two-level model does not depend on size of the valence space $\Omega$, at $V=1$ the BCS prediction is $T_{c}=0.455$. As seen from the figure this is not exactly correct, for a small 10-particle system the peak appears at about $T_{c}=0.35$, and only with the increase in the particle number the peak moves right to the BCS predicted value, thus confirming the BCS as an exact theory in the macroscopic limit.

In recent years analysis of poles in the complex temperature plane and the evolution of branches of these poles 


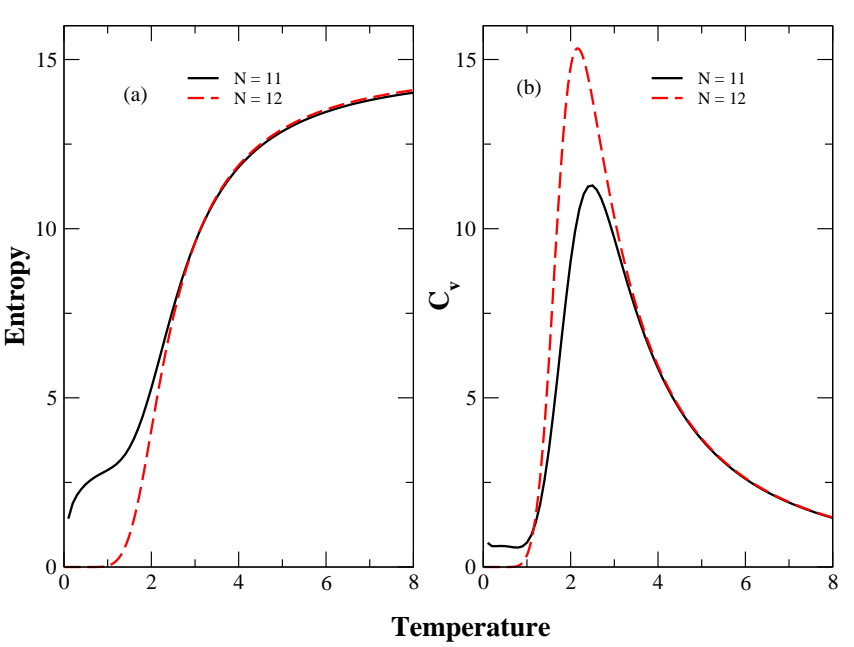

Figure 4: (Color online) (a) Entropy and (b) Specific heat as a function of temperature, for an odd and even number of particles and $V=1$.

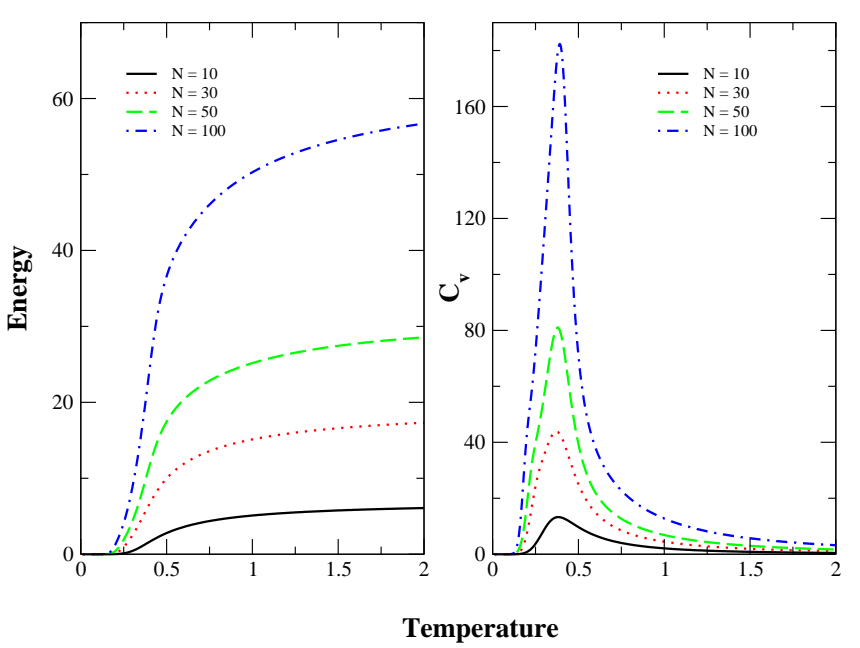

Figure 5: (Color online) (a) Energy and (b) Specific heat as a function of temperature, for $V=1$ and various number of particles $\mathrm{N}=10,30,50$, and 100 .

has attracted a lot of attention as a study and classification tool for mesoscopic phase transitions. The theory related to the distribution of zeros (DOZ) in fugacity of the grand canonical ensemble dates back to Yang-Lee [27, 28]. Later works [22, 23, 24] extended it the to the complex temperature plane of the canonical ensemble. The method of classifications of mesoscopic phase transitions, recently suggested in Ref. [11] is based on the distribution of zeros near the real axis. Some of the interesting questions such as whether the nature of the phase transition changes as a function of size have been studied with this approach. The first steps in the analysis of mesoscopic systems undergoing pairing phase transitions were done in Ref. [2, 62], the evolution of DOZ and com-

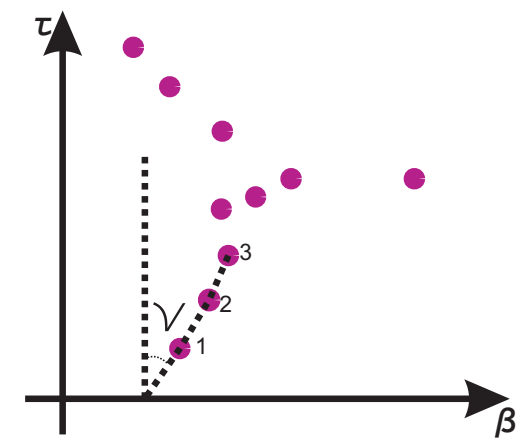

Figure 6: (Color online) The lowest branch of zeros computed for $\mathrm{N}=100$ and $\mathrm{G}=1.00$ is schematically shown.

parison with the thermal BCS for a two-level model can be found in Ref. [25].

In what follows we use the classification of phase transitions developed by Bormann et.al. [11]. We introduce complex temperature as $\mathcal{B}=\beta+i \tau$ and numerically seek a set of zeros $\mathcal{B}_{i}$ in the canonical partition function $\mathcal{Z}\left(\mathcal{B}_{i}, N\right)=0$, since the function is real the zeros appear in complex conjugate pairs and we can limit the region of consideration to $\tau \geq 0$. The product expansion of the partition function in terms of zeros using the Weierstrass theorem gives

$$
\mathcal{Z}(\mathcal{B})=\Omega \prod_{i}\left(1-\frac{\mathcal{B}}{\mathcal{B}_{i}}\right)\left(1-\frac{\mathcal{B}}{\mathcal{B}_{i}^{*}}\right) .
$$

The DOZ in the complex temperature plane for the twolevel system is shown schematically in Fig 6 .

The sets of zeros form branches [18, 25], in Fig[6 only the branch lowest to the real axis is shown. The size of the system determines the distance between neighboring zeros which in macroscopic limit becomes continuous. Phase transitions are associated with branches crossing the real axis. Indeed the zeros in the partition function appear as poles in thermodynamic variables; for energy or heat capacity we have from (29)

$$
\begin{gathered}
\langle E(\mathcal{B})\rangle=\sum_{i}\left(\frac{1}{\mathcal{B}_{i}-\beta}+\frac{1}{\mathcal{B}_{i}^{*}-\beta}\right), \\
C_{V}=\beta^{2} \sum_{i}\left(\frac{1}{\left(\mathcal{B}_{i}-\beta\right)^{2}}+\frac{1}{\left(\mathcal{B}_{i}^{*}-\beta\right)^{2}}\right) .
\end{gathered}
$$

In general, although there are no poles at the real axis, the derivative $d^{k}(\ln Z) / d \beta^{k} \sim \sum_{j}\left(\mathcal{B}_{i}-\beta\right)^{-k}$ may result in a divergent sum. As suggested in [11] the classification of phase transitions in the Ehrenfest sense can be extended to a smaller system by considering how the discrete roots of the phase transition branch approach the real axis. By labeling the roots in the phase transition branch starting from the closest one to real axis, see Fig. 6. the crossing angle can be given as

$$
\nu=\arctan \frac{\beta_{2}-\beta_{1}}{\tau_{2}-\tau_{1}} .
$$


The power law that expresses the congestion of roots as they approach real axis at $\tau \rightarrow 0$ determines the second parameter $\alpha$ as $\left|\mathcal{B}_{i+1}-\mathcal{B}_{i}\right| \sim \tau_{i}^{-\alpha}$.

The first order phase transition, which in thermodynamic limit appears as a discontinuity in the first derivative of the free energy corresponds to a vertical uniform approach of poles $\nu=0, \alpha=0$. In other cases the transition is of the second order for $0<\alpha<1$ or of a higher order if $\alpha>1$. This classification establishes a condition at which poles in sums of the form (30) and (31) accumulate a logarithmically divergent series. For a vertical approach, $\nu=0$ at the critical temperature the $\left|\mathcal{B}_{j}-\beta_{c r}\right| \sim j^{1 /(\alpha+1)}$ therefore $k$-th derivative of the partition function would lead to a divergent series if $k \geq \alpha+1$.

To find poles in the complex plane we developed a numerical technique that uses analiticity of the above functions. We first determine the number of roots in a desired region using a contour integral

$$
n=\frac{1}{2 \pi i} \oint\langle E(\mathcal{B})\rangle d \mathcal{B} .
$$

The line integration is fast and is done avoiding paths that go directly over the roots, this assures numerical stability and the real and integer result of Eq. (32) guarantees the accuracy. Once the number of roots is known we use a method in the spirit of the Laguerre's polynomial root finding technique 63. The problem is mathematically analogous to the two-dimensional problem of electrostatics. In the numerical method we converge to a given "charge" in the presence of the field from other "charges" which is modeled via multipole expansion using the analytically known derivatives of the "field strength". The found roots are sequentially removed, namely balanced by the "charge" of an opposite sign. Depending on the starting point and the density of roots, the numerical cancellation is not always perfect, and the same root may appear several times. Given that the total number of roots is known this problem is easily fixed by choosing a different starting point or by exploring a smaller region. In the calculations we stabilize the sum in the partition function by selecting scaling so that the largest term in the sum (22) equals unity.

A series of plots where evolution of poles in the complex temperature plane as a function of the pairing strength is shown in Fig. 7. The behavior of the heat capacity as a function of temperature for each case is shown below to highlight the phase transition point. With no pairing, $V=0$, the zeros are distributed along the two (almost) horizontal lines. Similar picture is seen at the pairing strength significantly below critical $\left(V_{c r}=0.5\right.$ at zero temperature from BCS). At about the critical strength, $V=0.4$, a noticeable bifurcation occurs with the lower branch evolving toward the real axis. As pairing strength increases, the branches move down and more branches becomes visible in our figures; in Fig. 7 we use the same temperature scale for all values of $V$. The lowest branch that approaches the real axis is associated with the phase transition. The latter is confirmed by the peak in the heat capacity that becomes sharper in cases with stronger pairing.

In Fig. 8 the dependence of the critical parameters $\nu$ and $\alpha$ on the pairing strength is addressed. Below the critical pairing strength the curves fluctuate, here, there is no phase transition and $\nu$ and $\alpha$ can not be interpreted as critical parameters. At a sufficiently strong pairing interaction, however, the behavior of the parameters stabilizes showing a second order phase transition.

\section{B. Grand Canonical ensemble}

The grand canonical ensemble is of a special importance in statistical mechanics, since the partition function for non-interacting particles, $\mathbf{Z}_{0}(\beta, \mu)$, is given by an analytical expression. The grand canonical partition function can be determined using the canonical one,

$$
\mathbf{Z}(\beta, \mu)=\sum_{N=0}^{\Omega} z^{N} \mathcal{Z}(\beta, N),
$$

where fugacity $z=\exp (\beta \mu)$ is introduced. For noninteracting Fermi particles

$$
\mathbf{Z}_{0}(\beta, \mu)=\prod_{1}\left(1+z \exp \left(-\beta \epsilon_{1}\right)\right),
$$

where $\epsilon_{1}$ is a single-particle spectrum. The above expression results, for example, in the commonly used form for the occupation numbers

$$
n_{i}=\left(1+\exp \left[\beta\left(\epsilon_{i}-\mu\right)\right]\right)^{-1} .
$$

The grand canonical approach and the use of the above Fermi distribution for small systems with a fixed number of particles is common, however may present serious problems. On the other hand, even for non-interacting particles computation of the microcanonical or canonical partition function is difficult [64]. Investigation of the mesoscopic limit where statistical ensembles may no longer be equivalent is of interest here.

The grand canonical ensemble is advantageous even when it comes to interacting systems, the partition function can be expressed via diagrammatic summation. In relation to pairing we mention here a method first proposed in Ref. [65], a more detailed discussion can be found in [66]. The full partition function for constant (or factorizable) pairing interaction can be obtained as

$$
\mathbf{Z}=\mathbf{Z}_{0} \int_{0}^{\infty} d t \exp (-Y(t))
$$

where function $Y(t)$ is

$$
Y(t)=t-4 \sum_{1>0} \ln \left[\frac{\cosh \left(\frac{\beta}{2} \sqrt{\epsilon_{1}^{2}+\frac{G t}{2 \beta}}\right)}{\cosh \left(\frac{\beta \epsilon_{1}}{2}\right)}\right] .
$$




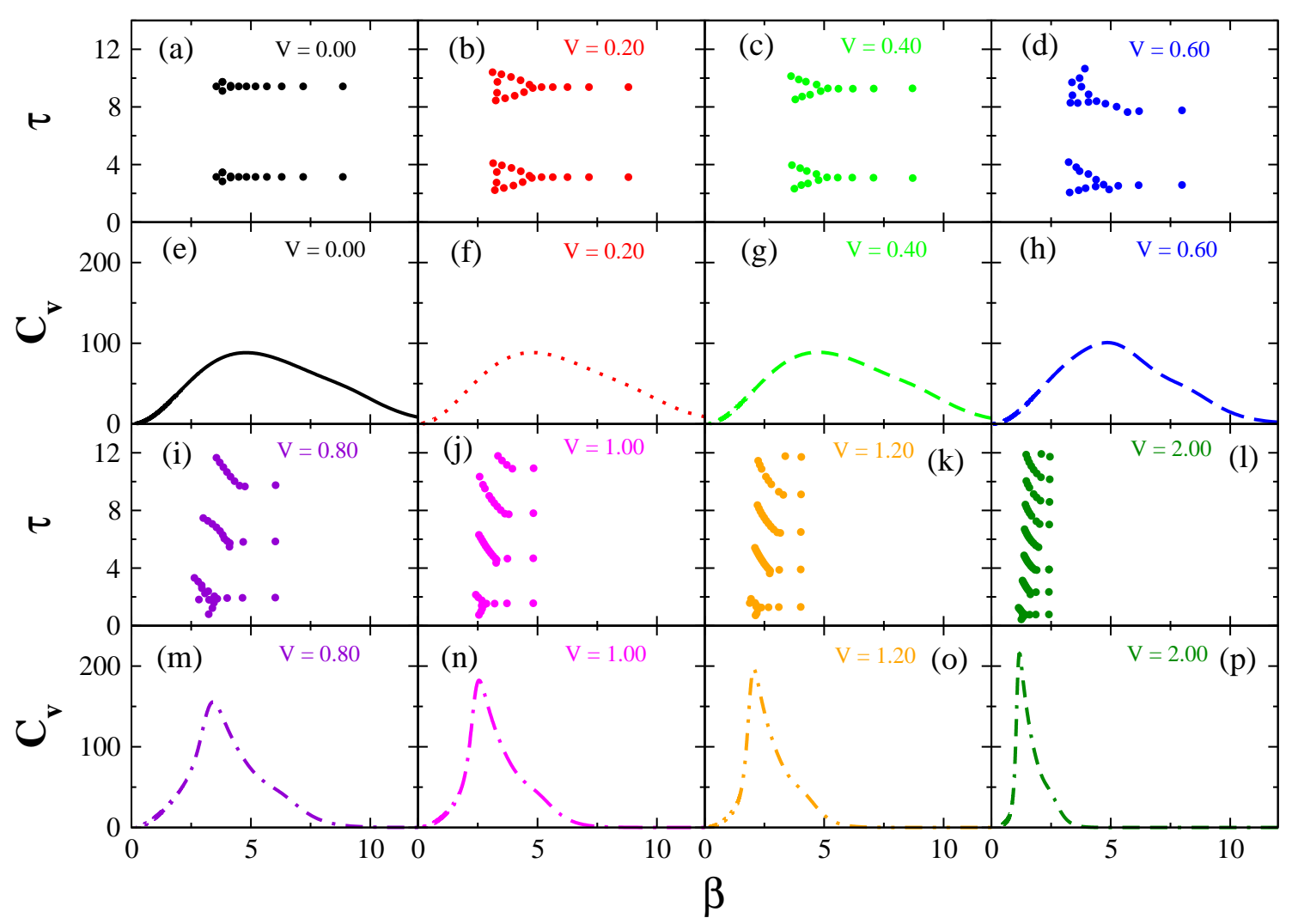

Figure 7: (Color online) Evolution of DOZ and $C_{v}$ in the complex temperature plane $\mathcal{B}=\beta+i \tau$ for $N=100$ particles in the half-occupied two-level system. There are number of poles near and exactly on the imaginary axis, they are of no interest to our discussion and are not shown. The poles for other systems are discussed in [2] and references therein, the interpretation and the nature of branches is discussed in [62], further in-depth exploration of the above model can be found in Ref. [25].

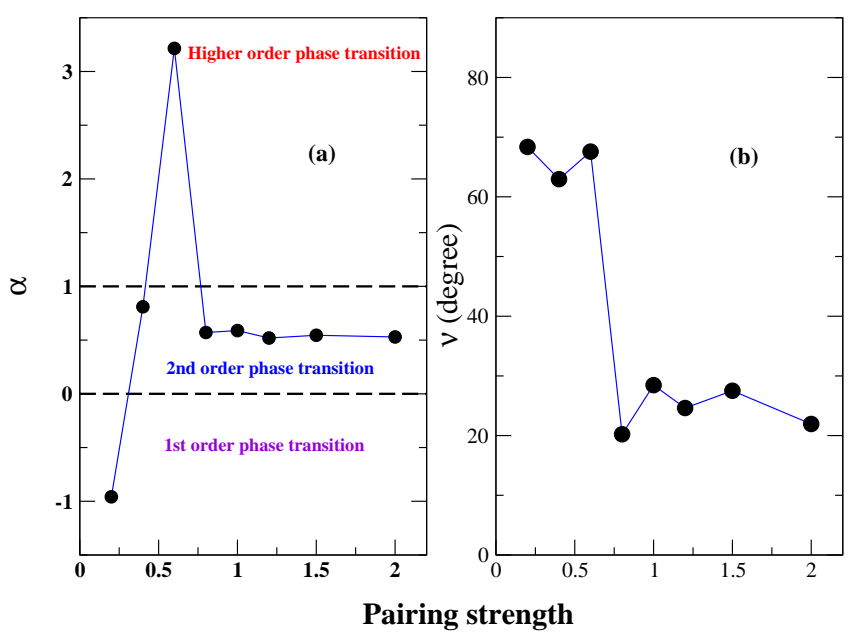

Figure 8: (Color online) Parameter of the phase transition for the two level system with $N=100$ particles as a function of pairing strength (a) $\alpha$ vs $V$ and (b) transition angle $\nu$ vs $V$.

The most straightforward saddle point approximation to the integral (34) leads to a saddle point $t_{s}$, which we express in terms of a gap parameter as $\Delta^{2}=G t_{s} /(2 \beta)$. Thus, the saddle-point equation becomes a familiar gap equation of thermal BCS (14), and the thermal BCS theory represents the lowest order approximation of the grand canonical expression in Eq. (34).

Various thermodynamic properties of the ladder system with 12 levels and 12 particles obtained with the exact calculation of the grand canonical partition function are shown in Fig. 9, the figure also includes comparisons with the corresponding curves from the canonical ensemble, where applicable. The fluctuations in the particle number as a function of temperature are shown in Fig. 9(a). The value of this quantity levels at about two particles, a similar uncertainty on a level of one pair is present in the BCS theory. The particle uncertainty relative to the system size $\sim 2 / N$ can be used to estimate the quality of the grand canonical ensemble in applications to particle-conserving mesoscopic systems. The results of comparisons between canonical and grand canonical ensembles for the entropy, excitation energy, and specific heat as a function of temperature are shown in Fig. 9(b)-(d). The difference is quite small, and is consistent with the level of error from the particle non-conservation. Further comparison is shown in Fig. 10 where the energy difference between canonical and grand canonical ensembles is plotted as a function of temperature. In the picket-fence model the discrepancy is noticeable, however 


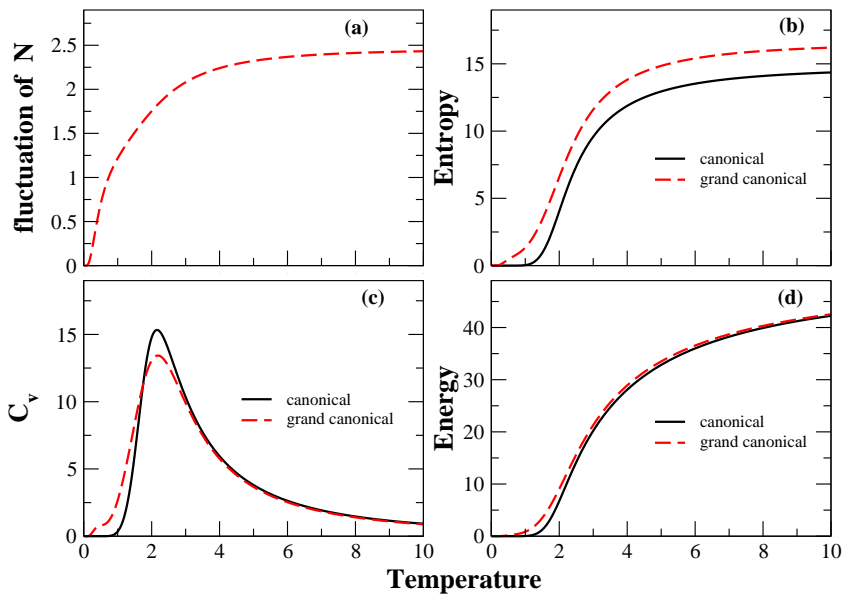

Figure 9: (Color online) Thermodynamic properties of the ladder system with 12 levels, 12 particles, and $V=1.00$ are shown as a function of temperature. The grand canonical ensemble is compared to the canonical. (a) Fluctuation in the number of particles in the grand canonical ensemble; (b) entropy; (c) specific heat; (d) excitation energy.

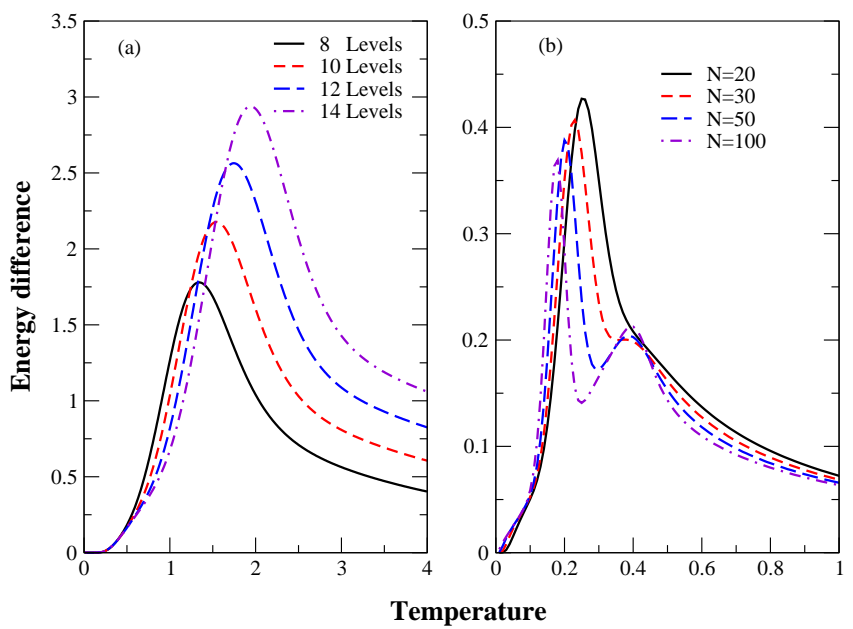

Figure 10: (Color online) The excitation energy difference between canonical and grand canonical statistical ensembles is shown as a function of temperature. Left panel corresponds to a ladder system and two-level model is on the right.

it becomes relatively small in the two-level case with a much larger number of particles. The difference peaks exactly at the temperature of the phase transition (in both cases $V=1$ ) where fluctuations are large. As seen in the two-level model for a large number of particles this region becomes narrow. Although for a ladder system the difference grows in the absolute scale, this behavior is associated with the extreme smallness of the system and the discrepancy per particle is still going to zero.

The zeros of the analytic continuation of the grand partition function into the complex plain of chemical potential are of a separate interest. We start by defining these points with the set of complex numbers $\mu_{i}$ that for a certain temperature satisfy the equation $\mathbf{Z}\left(\beta, \mu_{i}\right)=0$. There are some features to be stressed here. The number of principal roots $\mu_{i}$ is equal to the capacity of the fermion space $\Omega$. The grand canonical partition function (33) is an $\Omega$-th order polynomial in fugacity which leads to $\Omega$ roots in the chemical potential that can be found with the standard numerical techniques for polynomials. The methods discussed in the context of the canonical partition function are also useful in this case. As the size of the system grows the roots increase in number and may form branches that can approach the real axis. This describes the mesoscopic phase transition within the Yang-Lee picture [27, 28]. The accumulation of roots near the real axis, similarly to the canonical ensemble discussed earlier, represents a phase transition marked by the discontinuity in a certain order derivative of the grand canonical partition function with respect to chemical potential. This leads to the discontinuity in the pressure-volume diagram [67] and in the thermodynamic potential as a function of the particle number, namely condensation. Based on the well known properties of the Bose gas the appearance of such third order transition [68] could be a good evidence for the Bose-Einstein pair condensation. Whether with the increased pairing strength or in a certain limit of temperature the Cooper pairs become dynamically equivalent to bosons and form a condensate and if there is a crossover region is an interesting and important question [56, 69].

Before addressing the results of this study we discuss some of the expected features that can be inferred from the partition function (34). Within the BCS approximation the integral (34) is given by the single saddle point value

$$
\mathbf{Z}_{B C S}=\mathbf{Z}_{0} \exp \left(-\frac{2 \beta \Delta^{2}}{G}\right) \prod_{1>0}\left[\frac{\cosh \left(\frac{\beta}{2} e_{1}\right)}{\cosh \left(\frac{\beta \epsilon_{1}}{2}\right)}\right]^{4} .
$$

The Yang-Lee zeros of the above expression are zeros of the hyperbolic cosine and for each single particle energy $\epsilon_{1}$ an infinite series of roots labeled by integer $n$ can be obtained

$$
\mu=\epsilon_{1} \pm i \sqrt{\Delta^{2}+\pi^{2}\left(\frac{2 n+1}{\beta}\right)^{2}} .
$$

The evolution of DOZ of grand canonical ensemble in the complex plane of chemical potential for a fixed pairing strength $\mathrm{G}$ and various temperatures is shown in Figs. 11]13. In all of the plots only the principal branch of roots with $n=0$, Eq. (35), which is closest to the real axis is shown

In Fig. 11 a somewhat high pairing strength $V=1$ is selected so that at low temperature the system is well in the superconducting phase. The resulting zeros are located along the horizontal line consistently with Eq. (35). As the temperature increases the two lines of roots move apart deeper into the complex plane; this trend is again in agreement with (35), however the overall behavior of 
the roots is no longer regular. The critical temperature in this system (from the peak in heat capacity), is $T_{c}=0.38$ which coincides with a region where the behavior of DOZ changes. As seen from the figure there are no branches of substantial significance that cross real axis, indicating no phase transition.

The following Figs. 12 and 13 repeat the same study with weaker and stronger pairing. The findings are similar: at about critical temperature the DOZ changes from the two-line distribution, reflecting the BCS limit, to a more scattered set of roots moving away from the real axis at high temperature.

This exact calculation is consistent with the similar study 69]. At temperatures below critical and with strong pairing, Fig. 13, there are small symmetric branches of zeros that are directed toward the real axis (although never reach it), they do not appear to result in any transitional behavior and their significance is unclear. Our models lack the explicit spatial degree of freedom and it is likely that the BCS-BEC transition that reflects the change in the physical size of the Cooper pairs is simply impossible here.
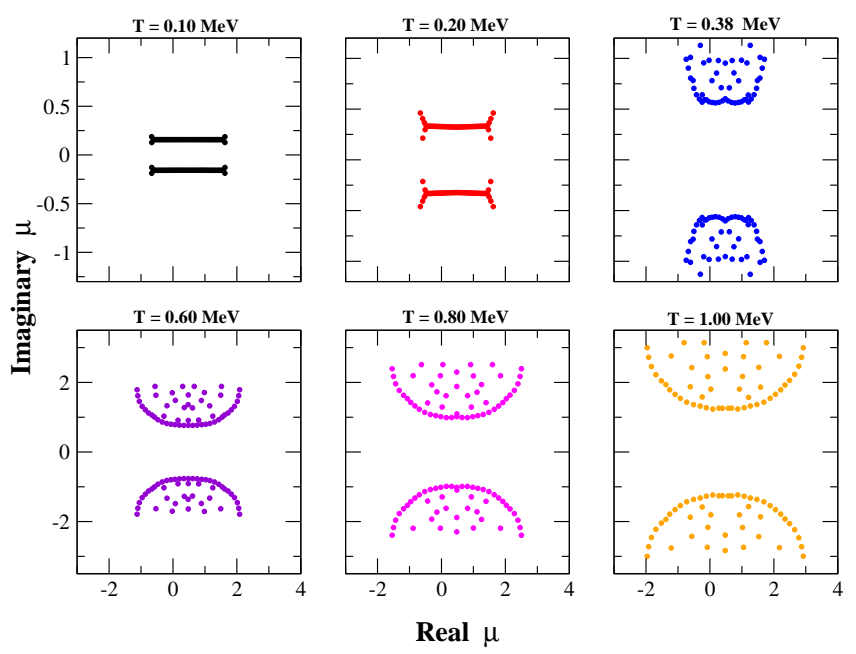

Figure 11: (Color online) The distribution of zeros (DOZ) of grand canonical ensemble in the complex chemical potential plane for a two levels system with $V=50$ and $V=1.00$ for various temperatures indicated. The $T_{c}=0.38$ for this system.

The grand canonical partition function (34) is useful for understanding DOZ in the complex temperature plane, although canonical and grand canonical ensembles are not fully equivalent. Balian and Langer [70] have determined that the zeros approach the real axis at an angle $\nu=\pi / 4$ and their density tends linearly to zero, $\alpha=1$, showing a second order transition.
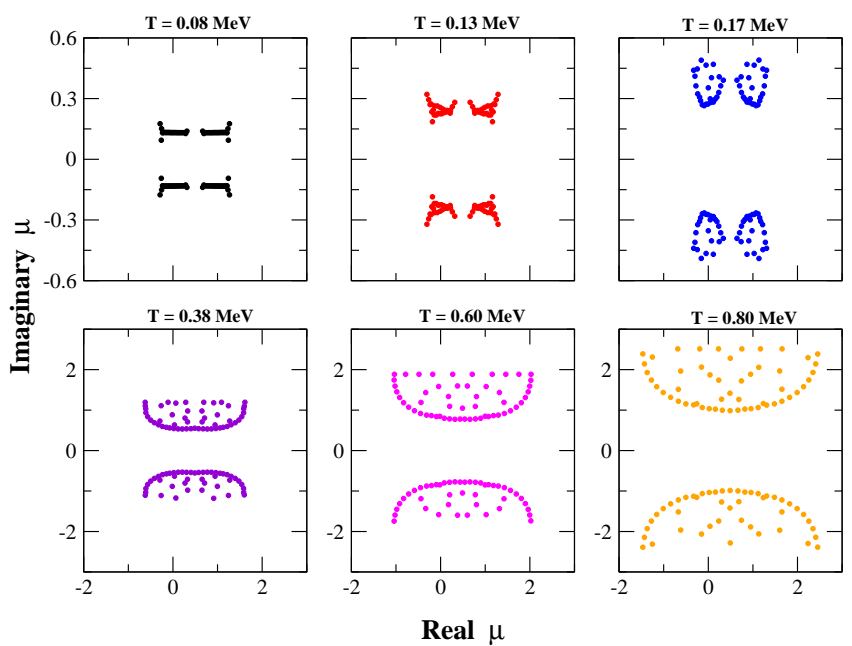

Figure 12: (Color online) Same as Fig. 11 except $V=0.5$ and corresponding $T_{c}=0.17$.
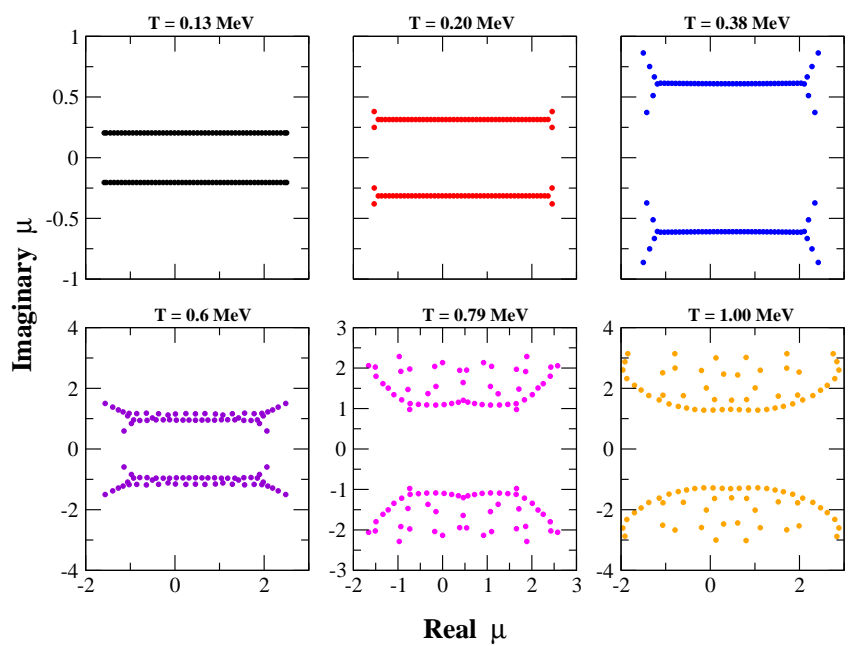

Figure 13: (Color online) Same as Fig. 11, except $V=2$ and thus $T_{c}=0.8$.

\section{Microcanonical ensemble}

The microcanonical ensemble is often assumed to be the most physically appropriate statistical treatment of a closed system. There is a number of theoretical works as well as direct experimental studies of nuclear thermodynamics in the microcanonical ensemble [19, 61, 71, 72]. The density of states (DOS) $\rho$ is the primary element in the approach. Regrettably, the formal definition given earlier (16) is not appropriate per se, the density of states as well as the normalization in the discrete spectrum requires some averaging energy interval. For most of this study we chose not to implement a traditional binning method substituting it by the propagator-type approach, where an artificially inserted small width $\sigma$ (the same for 
all states) results in the Lorentzian-type smoothing of every peak. The derivatives of the DOS are then calculated based on the analytic derivatives of the Lorentzian which provides an additional stability. With this procedure the DOS $\rho(E)$ is obtained. The averaging width $\sigma$ is an artificial parameter that introduces thermodynamic averaging, the results may strongly depend on this parameter when it is smaller than the average level spacing. This parameter is not necessarily a disadvantage, to the contrary, it allows us to zoom at the energy scale of interest. Within this work we select $\sigma=0.5-1.0$ in single-particle level spacing units. This is most reasonable micro scale and can be compared with the resolution scale of the canonical ensemble where energy fluctuations are at about 10. The $\sigma$ interval versus level spacing can be interpreted as the number of states needed to obtain a statistically equilibrated value for observable quantities, in the limit of quantum chaos a single state is sufficient [49, 73], on the other hand as discussed below pure pairing due to seniority conservation is poorly equilibrated and many states must be included. The latter fact influenced our choice of $\sigma$.

The entropy in the microcanonical ensemble is

$$
S(E)=\ln \rho(E),
$$

and the temperature can be defined as

$$
T(E)=\left(\frac{\partial S(E)}{\partial E}\right)^{-1}
$$

which does not depend on the normalization that is used for the DOS.

In Fig. 14 the temperature is shown as a function of excitation energy for all three ensembles. The microcanonical curve with $\sigma=1$ shows several low-lying peaks that can be identified with the pair breaking [17, 20, 72]. The seniority is a conserved quantum number for pure pairing interaction, nevertheless these peaks survive in the presence of all interactions as was shown in Ref. [48, 71, 74]. The corresponding oscillations in the heat capacity and especially the regions where this quantity is negative can be associated with the paired to normal phase transition which takes place in the pair-by-pair microscopically fragmented process. The canonical and grand canonical ensembles due to thermalization created by the heat bath smooth out these microscopic features into a single phase transition, and importantly, the same can be done in the microcanonical treatment by choosing a large averaging interval $\sigma$. Already at $\sigma=5$, which is about half the energy fluctuation in the canonical ensemble, the peaks in Fig. 14 disappear and microcanonical approach becomes similar to canonical and grand canonical.

The macroscopic limit of the microcanonical ensemble is considered in Fig. 15 where entropy as a function of excitation energy in the two-level system for various $N$ is shown. The comparison of microcanonical, canonical and grand canonical treatments indicates that they become identical in the thermodynamic limit. The discrepancy

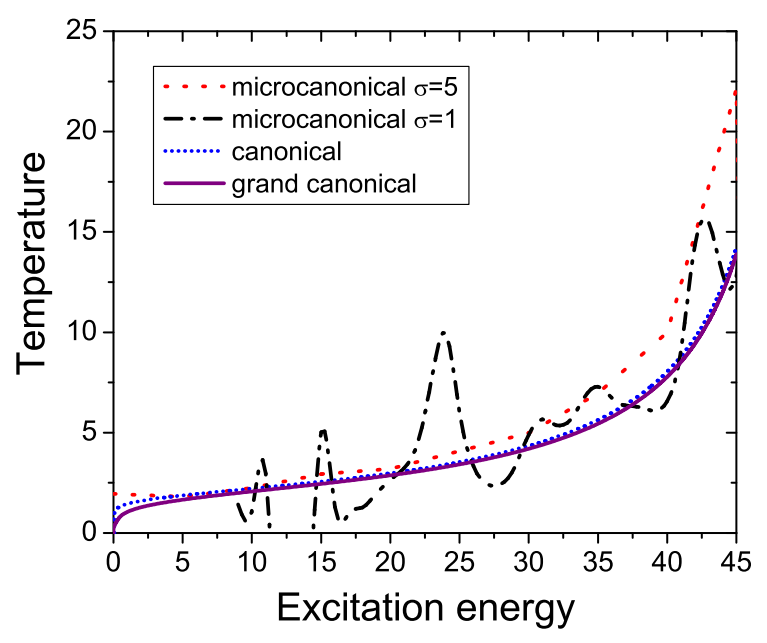

Figure 14: (Color online) Temperature as a function of energy in three different statistical treatments: canonical, grand canonical and microcanonical. The ladder system with 12 levels, 12 particles and $V=1.00$ is used for this study. The results for the microcanonical ensemble are plotted with two different choices of energy window $\sigma=1$ and 5 .

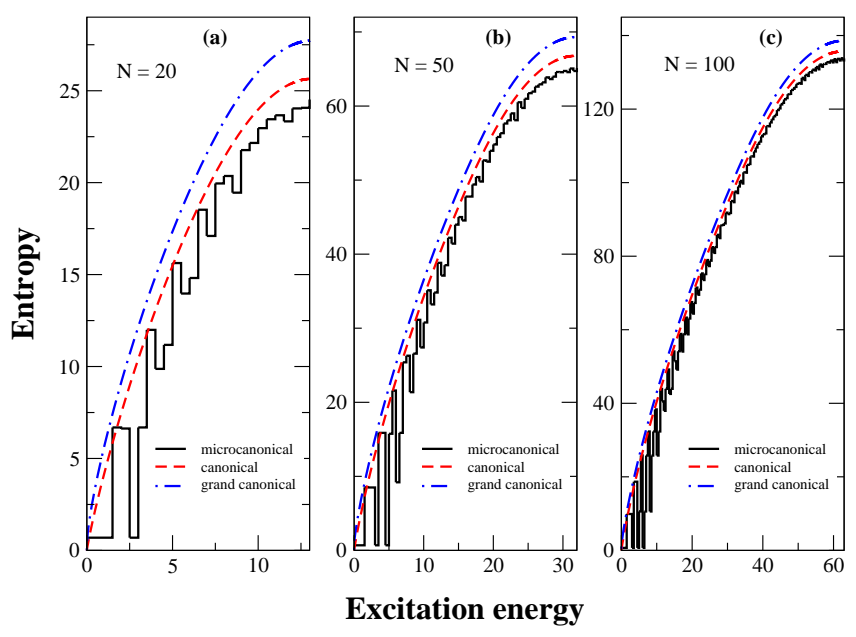

Figure 15: (Color online) Comparison of entropy as a function of excitation energy for the two-level system in three thermodynamic ensembles ( $\sigma=0.5$ in microcanonical). Pairing strength $V=1.00$. (a) For $\mathrm{N}=20$ particles; (b) $\mathrm{N}=50$ particles; (c) $\mathrm{N}=100$ particles.

at high energy is related to the finite space where in the microcanonical case the density of states becomes zero once the energy exceeds the maximum possible value for the model space. The model space limitation is a natural cut-off for all ensembles at high energy.

In contrast to the canonical and grand canonical ensembles where thermalization is provided by the external heat bath the thermalization is a serious question in the microcanonical treatment [75, 76]. It has been noted in 
Refs. [20, 54, 74] that pairing interactions do not provide sufficient thermalization. Particle-particle interactions of the pairing type only are not sufficient to fully mix states and thermodynamically equilibrate the system. Temperature determined microscopically (37) is inconsistent with the one that comes from the occupation numbers of individual single-particle levels see Ref. $[20,54,74]$. This property of pairing makes the microcanonical treatment special. The question of thermalization in systems with pure pairing is rather academic; as it has been shown in [20] and references therein, at an arbitrary weak non-pairing interactions the equilibration is immediately restored. The significant role of non-pairing interaction was explored in recent work [77]. The magnetic field discussed below can also provide the needed thermalizing effect. The sharp differences in the statistical approaches seen in Fig. 14 suggest to look for an alternative treatment and tracking of the transitional behavior which would not introduce the heat bath, energy averaging, or the particle number uncertainty but at the same time is statistically equilibrated. The Invariant Correlational Entropy in the next section provides a tool that satisfies this criteria.

\section{INVARIANT CORRELATIONAL ENTROPY}

The Invariant Correlational entropy (ICE) [57, 59] is a powerful method that allows phase transition features to be explored on a quantum mechanical level. Expanding the formal definitions of Sec. IV the ICE for an individual eigenstate $\alpha$ is computed by averaging the density matrix over the interval of pairing strength

$$
I^{\alpha}=-\operatorname{Tr}\left(\overline{\rho^{\alpha}} \ln \overline{\rho^{\alpha}}\right), \quad \overline{\rho_{k k^{\prime}}^{\alpha}}=\overline{\langle k \mid \alpha\rangle\left\langle\alpha \mid k^{\prime}\right\rangle},
$$

here $k$ is a basis state. The final result due to the trace operation is basis independent. In Fig. 16 we show the invariant correlational entropy for all states in the paired $N=10$ two-level system as a function of the excitation energy of the corresponding state. The ICE fluctuates from state to state and the curve shown has been smoothed. The enhancement of the ICE in the region between 0 and 6 energy units of excitation signals a transitional behavior. Indeed, a lower figure that shows the specific heat as a function of energy for the same system in the canonical ensemble reveals a consistent trend. The advantage of the ICE is that unlike canonical (or grand canonical) ensemble it needs no heat bath and maintains an exact particle conservation; on the other hand, it is not prone to equilibration and thermalization issues since those are established by the fluctuations of the pairing strength.

\section{PAIRING AND MAGNETIC FIELD}

The presence of magnetic field is known to influence the physics of the pairing state and the pairing phase
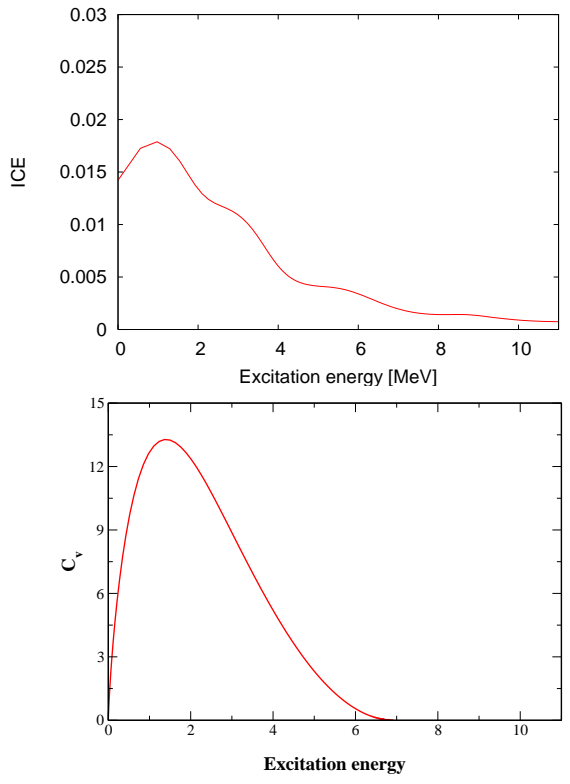

Figure 16: (Color online) Invariant correlational entropy (ICE), upper panel; and the specific heat in the canonical ensemble, lower panel, are shown as a function of excitation energy for the two-level system with $\mathrm{N}=10$ particles and $V=1$.

transition. In this section we extend our study by showing the changes to the results brought by the presence of the field. The Hamiltonian to be considered here is

$$
H_{B}=H-\mathrm{g} J \cdot \mathbf{B}
$$

where $J$ is the angular momentum of the state and $B$ is the magnetic field. Without loss of generality we choose units of the magnetic field so that the gyromagnetic ratio $\mathrm{g}=1$. The introduction of the magnetic field does not require a new diagonalization of the Hamiltonian. All eigenstates shift in energy according to their magnetic quantum number $M$, with the quantization axis along the field $\mathbf{B}$. The problem outlined with the Hamiltonian (38) is identical to the cranking model of rotating nuclei where $H_{\omega}=H-J \cdot \omega$ with $\omega$ representing rotational frequency. Thus, a reader interested in rotations should understand "magnetic field" as a "rotational frequency".

In the case of a single particle on one level the magnetization is a textbook example

$$
\langle M\rangle=\frac{1}{2}\left[(2 j+1) \operatorname{coth}\left(\frac{1}{2}(2 j+1) x\right)-\operatorname{coth}\left(\frac{x}{2}\right)\right] .
$$

The spin fluctuation $\chi(\beta, B)=\left\langle M^{2}\right\rangle-\langle M\rangle^{2}$, related to spin susceptibility, is given by

$$
\chi=\frac{1}{4}\left(\operatorname{csch}^{2}\left(\frac{x}{2}\right)-(2 j+1)^{2} \operatorname{csch}^{2}\left(\left(j+\frac{1}{2}\right) x\right)\right) .
$$

Here $x=g B / T$. The generalization of these results to the cases with many levels is straightforward.

In our study below we assume that the degeneracy in the single-particle spectrum is due to the corresponding 
value of the angular momentum $j=\omega-1 / 2$ for the twolevel model and $j=1 / 2$ for all levels in the ladder system. For each seniority we deduce the number of states with certain angular momentum which in turn allows us to calculate statistical partition functions. The analytically computed summations over the magnetic quantum numbers such as in Eqs. (40) and (39) speed up the calculations.

The destruction of the superconducting state occurs because of the two somewhat related phenomena. A magnetic field causes the breaking of superconducting pairs due to the lowering in energy of the spin aligned states. The estimate for the critical field in this case can be obtained by comparing the energy of the paired ground state with the seniority $s=2$ aligned spin state with spin $J$. The latter is by $2 \Delta$ higher in energy at zero field and pairing becomes unfavorable when magnetic field exceeds $B_{c r}=2 \Delta /(\mathrm{gJ})$. In our models, equations such as (12) or (15) can be used for an estimate. The second reason is the change in the energy of the normal state reflecting the Pauli spin paramagnetism. In our models (half-occupied for the two-level case) the field above $B_{c r}=\Delta \epsilon /(2 \mathrm{~g} j)$ will promote the particle-hole excitations across the gap between the single particle levels. It has been suggested [78] and experimentally confirmed, for example in Ref. [79], that due to these phenomena a sufficiently strong magnetic field could change the transition type from second to first order. The situation can be quite complex in mesoscopic systems where even without the field the classification can be somewhat difficult.

The features of a 20-particle half-occupied two-level system are shown in Fig. 17 as a function of temperature for a set of different values of magnetic fields. Except for $B=0$, for all curves in this figure the field exceeds both of the above critical values $\left(B_{c r} \sim 0.1\right)$. The behavior of the heat capacity illustrates the disappearance of the phase transition for all field strength shown, except for $B=0$ where a sharp peak is present. The average magnetization Fig. 17(c) is exactly zero in the absence of field and for high fields starts almost at the saturation. With increased temperature magnetization drops down.

The set of fields below critical is shown in the next figure (18). The behavior of the magnetic spin fluctuations is regular at $B=0$, at weak fields this quantity exhibits a sharp peak at low temperatures, for strong fields the regular behavior is again restored. The same peak is present in the spin susceptibility curve which is only by a factor of $\beta^{2}$ different from $\chi$. The critical behavior is associated with the corresponding behavior of the magnetization, see Figs. 19 and 20. The peak in the heat capacity is reduced and shifted to lower temperature, showing that at non-zero field a superconductor has lower critical temperature.

These results are quite robust. In Fig. 19 we show the same study repeated for the ladder model. The magnetization (upper left panel in Fig. 19) is zero for no field, it shows a peak in the region of the field strengths that corresponds to competition between the paired state

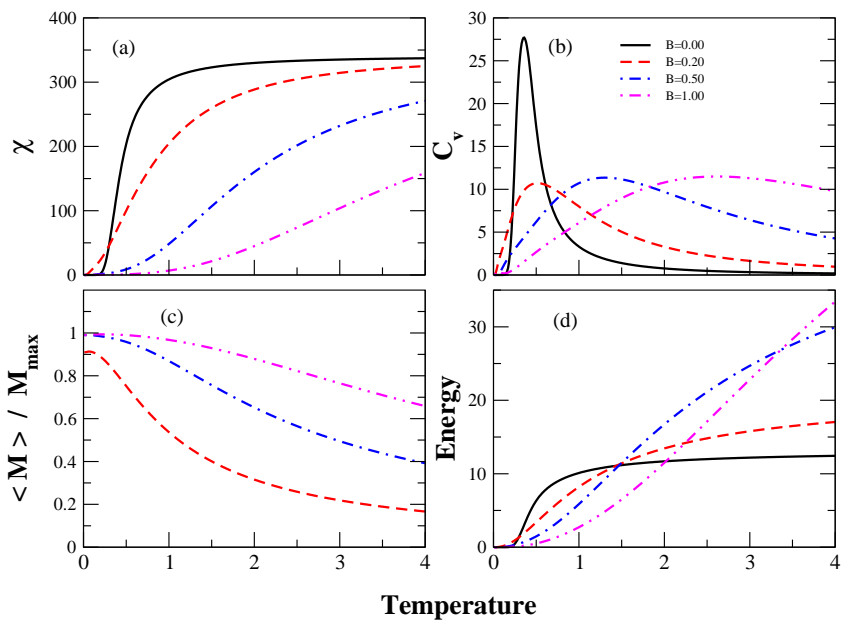

Figure 17: (Color online) Thermodynamics of the two level system with $N=20$ and $V=1.00$ in the magnetic field. The (a) spin fluctuations $\chi$; (b) specific heat; (c) magnetization (ratio to the maximum possible value); and (d) energy are shown as functions of temperature for different field strengths. For this model $T_{c r}=0.46$ at $B=0$.

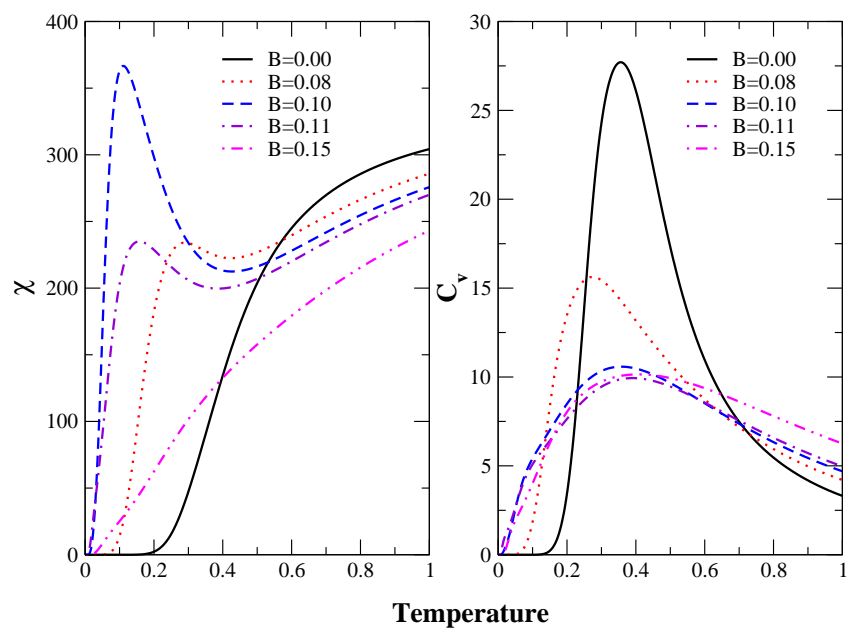

Figure 18: (Color online) The same system as in Fig. 17 but concentrating on the low magnetic fields. (a) spin fluctuations, (b) specific heat.

and magnetic orientation ( $B=5$ and 7 curves), and finally at strong fields and low temperature it saturates to $\langle M\rangle=6$, the maximum alignment state. The spin fluctuations, shown on the left lower panel, at zero temperature and no field, are consistent with the typical results [80]. The sharp peak at magnetic fields below critical again reflects the transition associated with magnetic alignment. The emergence of the peak that in thermodynamic limit would become a discontinuity in the otherwise continuous curve at $B=0$ can be interpreted as the change in the type of the phase transition. The evolution of the transition point as a function of temperature is seen in the heat capacity curve which again shows lowering of the tran- 


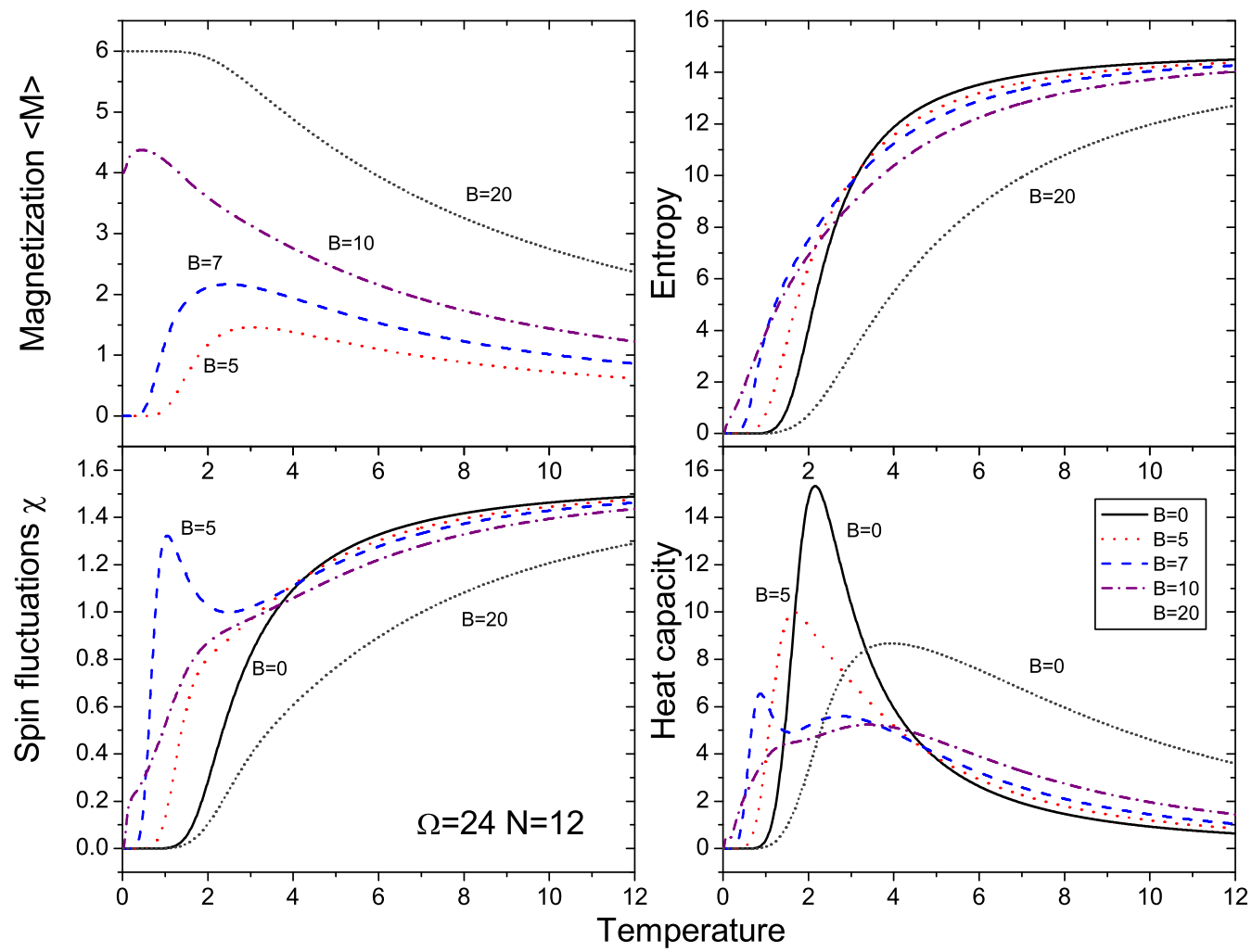

Figure 19: (Color online) Thermodynamics within the canonical ensemble of a 12-level ladder system with $N=12$ particles in the magnetic field. The pairing strength is $V=1$. The panels include plots of magnetization, entropy, magnetic susceptibility and specific heat as a function of temperature. The curves correspond to five different values of the magnetic field $\mathrm{B}=0,5,7$, 10 and 20.

sition temperature with the increased field. The finding can be summarized as the existence of two critical values for the magnetic field first one that corresponds to the change in the nature of the normal-to-superconducting transition; and at the second, higher value of the field, the paired state is no longer supported. The change in the nature of the phase transition is best seen in the spinsusceptibility curve which at low fields has no peak and acquires a peak consistent with the peak in heat capacity at higher values of the magnetic field. We associate this behavior with the analogous situation in the macroscopic superconductor where the second order phase transition becomes first order in the non-zero magnetic field.

Recently, sharp differences between the systems with odd and even particle numbers have been discussed in the literature [62, 80]. We address this in Fig. 20 where we show the same study as in Fig. 19 but with $N=11$ particles. The primary difference between these results is that the ground state is degenerate and both magnetization and entropy are non-zero at low temperature and low field. Otherwise the results are almost identical. We conducted similar calculations for a two-level model with
$N=19$ particles but decided not to show the results because the difference between $N=20$ and $N=19$ is almost impossible to notice (except for the entropy and magnetization at zero temperature and zero field).

The presence of the external magnetic field certainly has an effect on the distribution of zeros in the complex temperature plane. This evolution is explored in Fig. 21 where the motion of roots is traced as the magnetic field is increased in small increments. The initial distribution of roots is connected with a line marked by $B=0$. The second line connects the roots at $B=0.01$. The gradual rotation of the branch responsible for the phase transition is seen, which eventually, at high fields, no longer orients the roots toward the real axis. Based on a similar picture but for a large system with $N=100$ particles that was studied earlier in Fig. 22, we show the change in the critical parameters associated with this motion in the presence of the field. Interestingly, both $\alpha$ and angle $\nu$ are approaching zero which marks the change in the phase transition type from second to first order. Unfortunately, the zero $\alpha$ is not reached since the field strength becomes larger then the critical (here $B_{c r} \sim 0.01$ ) and the paired 


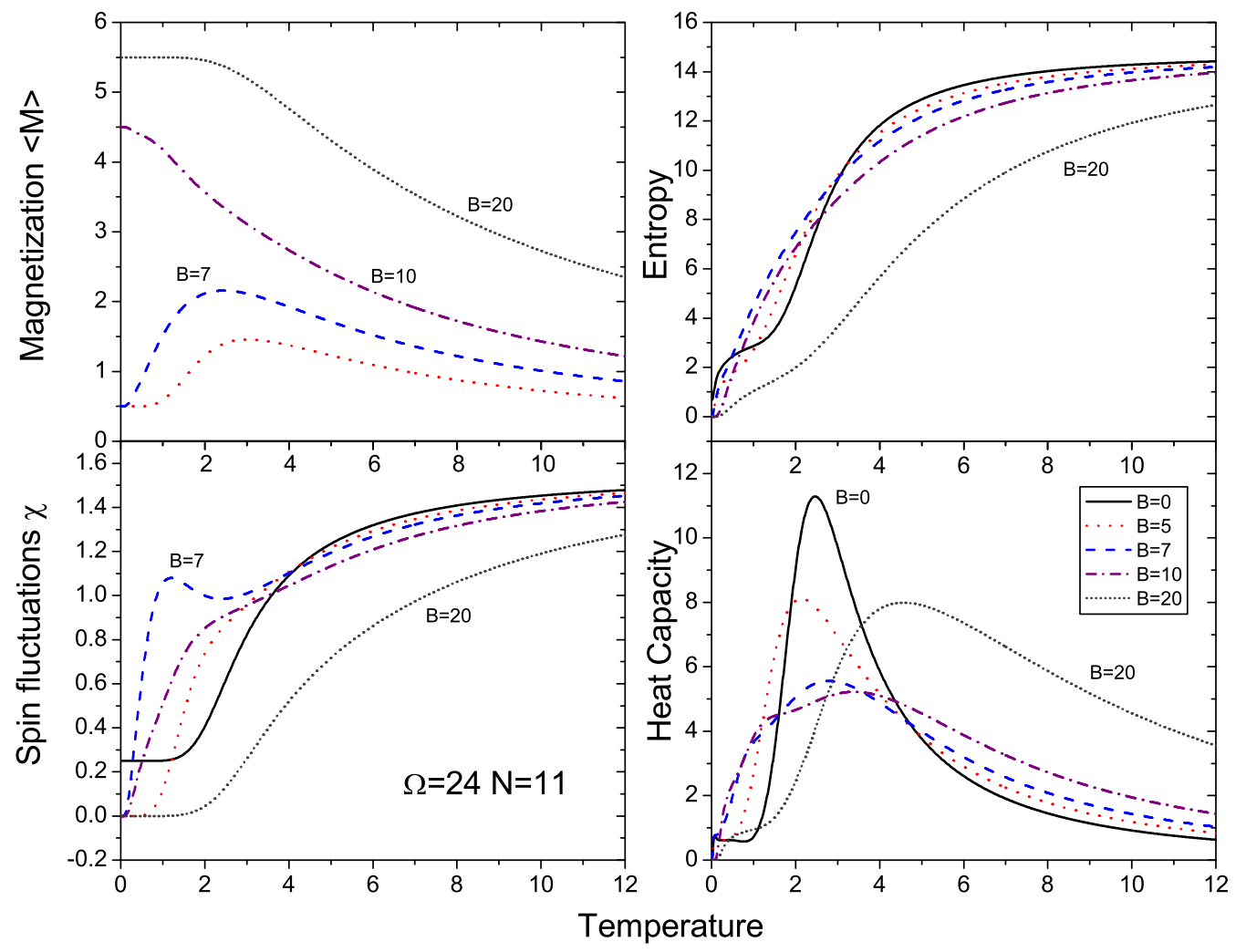

Figure 20: (Color online) Thermodynamics within the canonical ensemble of a 12-level ladder system with 11 particles in the magnetic field. The pairing strength is $V=1$. The panels include plots of magnetization, entropy, magnetic susceptibility and specific heat as a function of temperature. The curves correspond to five different values of the magnetic field $\mathrm{B}=0,5,7,10$ and 20 .

phase disappears. The plot in Fig. (22) is ended at this point since it is becomes impossible to identify a branch of roots relevant to the phase transition.

Finally we mention a thermalizing role of the magnetic field which breaks high degeneracies of states; and as well as changing the phase transition globally it reduces the number of individual pair breaking transitions seen in the microcanonical treatment. In Fig. 23 the entropy in the microcanonical ensemble is plotted as a function of excitation energy for different strengths of the external magnetic field. The number of peaks associated with the pair breaking is ten at zero field and this number becomes smaller as the magnetic field gets stronger, simply because of the pair alignment. Thermal equilibration and the equivalence of ensembles are seen in the following Fig. 24. In contrast to the $B=0$ case in Fig. 14, the difference between canonical and microcanonical ensembles disappears at the magnetic field near critical.

\section{SUMMARY CONCLUSIONS AND OUTLOOK}

Using an exact solution to the pairing problem in a picket-fence and two-level systems we addressed different views on the pairing phase transition, pair breaking, thermalization, behavior in the magnetic field or, equivalently, rotation in the framework of the cranking model. We present a comprehensive study analyzing paired systems with various tools and methods ranging from the BCS treatment to different thermodynamic approaches, including invariant correlational entropy and zeros of the partition functions in the complex plane of temperature and chemical potential.

We found the microcanonical, canonical, and grand canonical thermodynamic approaches to be different when applied to small systems, although as expected they are consistent in the macroscopic limit. The grand canonical and canonical treatment are surprisingly close to each other even in the cases with a relatively small particle number. We find the microcanonical treatment to be the 


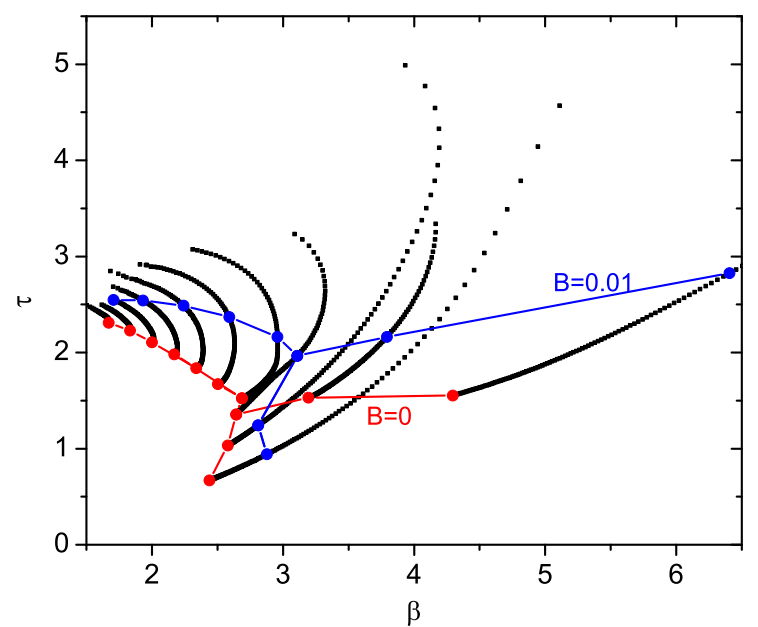

Figure 21: (Color online) The distribution of zeros without and with the presence of external magnetic field for two level system $\mathrm{N}=60$ and $\mathrm{G}=1.00$.
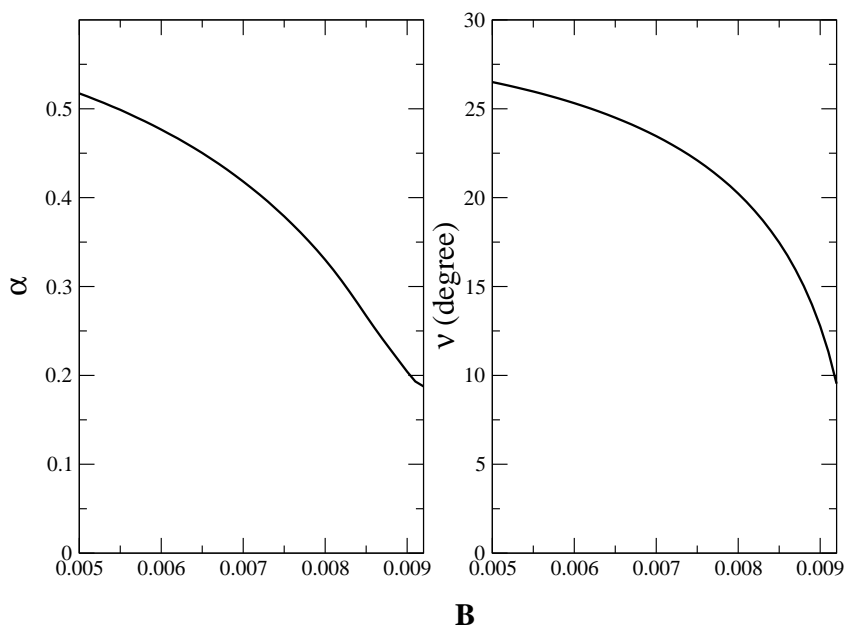

Figure 22: The evolution of critical parameters $\alpha$ and $\nu$ as a function of applied magnetic field $B$ in system with $N=100$ particles and $V=1.00$

most adequate approach for closed small systems, it allows for both global and relatively local, in terms of the energy scale, consideration of the statistical properties. The averaging energy window needed for determination of the density of states gives a broad freedom to the microcanonical approach. The corresponding energy fluctuation in canonical and grand canonical treatments is too large in small systems and smooths out many significant statistical features such as individual pair breaking observed in experiment [71]. In thermally equilibrated systems the energy window can be as small as few times the level spacing since in the full quantum chaos an individual state is a representative of the surrounding statistical

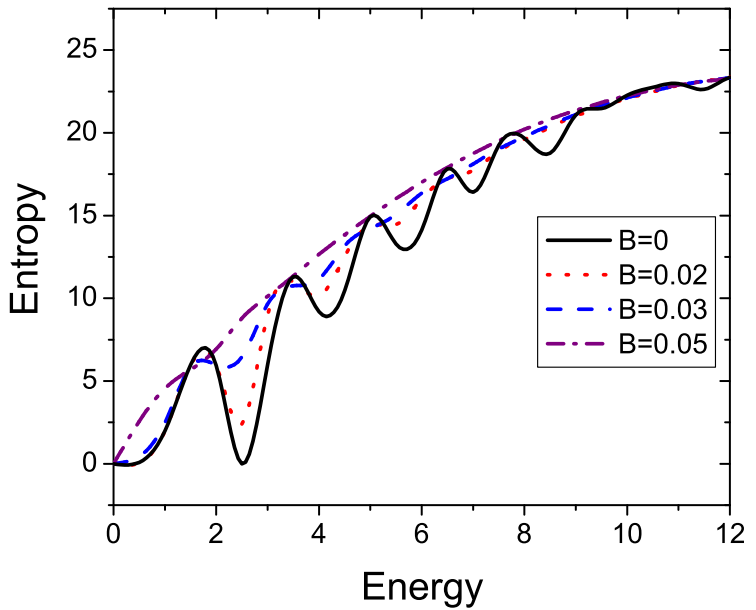

Figure 23: (Color online) Entropy in the microcanonical picture is shown as a function the excitation energy for several values of the magnetic field below critical. The two-level system with 20 particles and $V=1$ pairing strength was used.

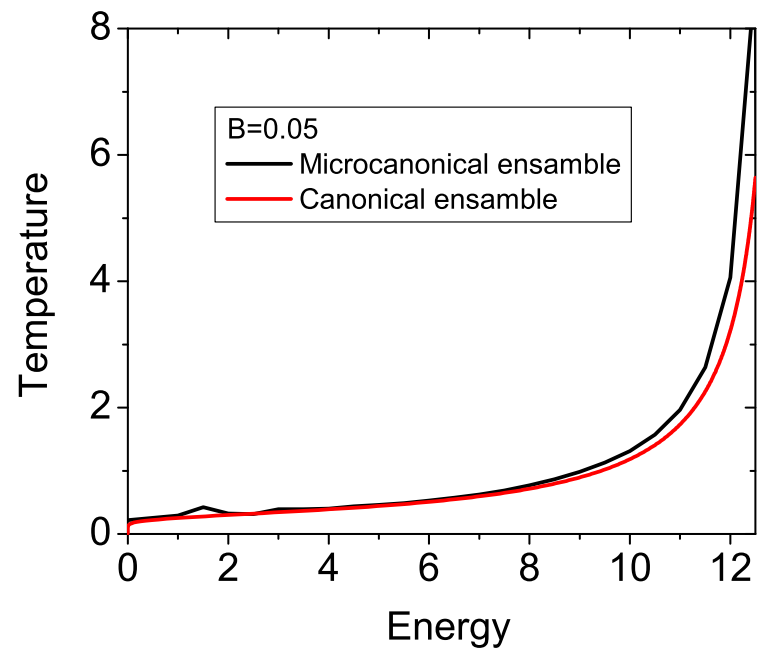

Figure 24: (Color online) The same system as in Fig. 23. the temperature as a function of energy is compared in microcanonical and canonical ensembles at the magnetic field strength $B=0.05$. The difference at high energy is due to a finite model space.

properties [73]. The idealization of interaction limiting them to pairing only represents a dangerous problem: the pairing forces exclusively cannot establish full equilibration, this necessitates a larger thermodynamic energy window.

The Invariant Correlational Entropy that relies on fluctuations in the pairing strength as a source of equilibra- 
tion appears to be a powerful statistical tool, capable of exploring most of the features inherent separately to microcanonical, canonical and grand canonical ensembles. This tool is particularly important in identifying phase transition regions in mesoscopic systems.

Turning to the properties of the phase transition we found as mentioned above the microcanonical treatment to be distinct from other ensembles. In thermodynamic limit, however, all treatments agree. In the further study of phase transitions we discussed the distribution of zeros of the canonical partition function in the complex temperature plane. We developed and implemented a numerical method for counting and finding all of the complex roots in a given region. In agreement with the earlier findings [2, 25, 62] we observe branches of roots and study the properties of the one that approaches the real axis. The behavior of the roots is consistent with the second order phase transition as classified in [23, 24] and confirms similar macroscopic results [70].

The recent interest to the crossover region between superconducting pairing and Bose-Einstein condensation of pairs prompted us to consider the potential condensation by looking for zeros of chemical potential in the grand canonical partition function. The presence of such zeros near real axis would hint on the condensation phenomenon. We did not find significant branches of roots evolving toward the real axis, and no critical behavior was observed in thermodynamics. It is likely that our model with no explicit spatial degrees of freedom is not appropriate for these questions.

The last chapter of our work is devoted to interesting study of the mesoscopic phase transition in the presence of magnetic field. It is fully equivalent to rotations within cranking model. We found that there is a resemblance between observed mesoscopic properties and those known in the macroscopic physics of superconductors. At low field the normal and superconducting phases are separated by the second order phase transition. In the next region of higher magnetic field the normal and superconducting phases are separated by the transition of a different nature associated with a simultaneous peak in spin susceptibility end enhanced spin fluctuations. Finally, at even higher fields a superconducting state is not supported at all. We conjecture that this behavior is a mesoscopic manifestation of the second to first order change in the transition type known in the thermodynamic limit. We also traced the evolution of zeros in the canonical partition function as a function of magnetic field. We found that the classification of transition type as suggested in Ref. [11] is consistent with the above argument.

\section{Acknowledgments}

The authors acknowledge support from the U. S. Department of Energy, grant DE-FG02-92ER40750. We are grateful to $\mathrm{V}$. Zelevinsky for collaboration and invaluable advice on many topics presented in this work. We wish to think A. Schiller, T. Døssing and P. Ipsen for useful comments and references.
[1] L. Cooper, Phys. Rev. 104, 1189 (1956).

[2] D. J. Dean and M. Hjorth-Jensen, Rev. Mod. Phys. 75, 607 (2003), nucl-th/0210033.

[3] P. Borrmann and J. Harting, Phys. Rev. Lett. 86, 3120 (2001).

[4] F. Braun and J. von Delft, Phys. Rev. Lett. 81, 4712 (1998).

[5] F. Braun and J. von Delft, Phys. Rev. B 59, 9527 (1999).

[6] J. von Delft, Ann. Phys. 10, 219 (2001).

[7] J. Harting, O. Mulken, and P. Borrmann, Phys. Rev. B 62, 10207 (2000).

[8] C. Kawabata and M. Suzuki, J. Phys. Soc. Jap. 28, 16 (1970).

[9] M. Suzuki, Prog. Theor. Phys. 41, 1438 (1969).

[10] A. Belic, D. J. Dean, and M. Hjorth-Jensen, Nucl. Phys. A731, 381 (2004), nucl-th/0309079.

[11] P. Borrmann, O. Mulken, and J. Harting, Phys. Rev. Lett. 84, 3511 (2000).

[12] H. Chen, J. R. Brownstein, and D. J. Rowe, Phys. Rev. C 42, 1422 (1990).

[13] P. Chomaz and F. Gulminelli, Nucl. Phys. A749, 3c (2005).

[14] C. Bahri, D. J. Rowe, and W. Wijesundera, Phys. Rev. C 58, 1539 (1998).

[15] F. Barranco, R. A. Broglia, G. Gori, E. Vigezzi, P. F.
Bortignon, and J. Terasaki, Phys. Rev. Lett. 83, 2147 (1999).

[16] F. Barranco, P. F. Bortignon, R. A. Broglia, G. Colo, and E. Vigezzi, Eur. Phys. J. A 11, 385 (2001).

[17] A. Schiller, A. Bjerve, M. Guttormsen, M. Hjorth-Jensen, F. Ingebretsen, E. Melby, S. Messelt, J. Rekstad, S. Siem, and S. W. Odegard, Phys. Rev. C 63, 021306(R) (2001).

[18] A. Schiller, M. Guttormsen, M. Hjorth-Jensen, J. Rekstad, and S. Siem, Phys. Rev. C 66, 24322 (2002).

[19] A. Schiller, E. Algin, L. A. Bernstein, P. E. Garrett, M. Guttormsen, M. Hjorth-Jensen, C. W. Johnson, G. E. Mitchell, J. Rekstad, S. Siem, et al., Phys. Rev. C 68, 054326 (2003).

[20] V. Zelevinsky and A. Volya, Phys. At. Nucl. 66 (2003).

[21] W. Bestgen, S. Grossman, and W. Rosenhau, J. Phys. Soc. Jap. S 26, 115 (1969).

[22] S. Grossman and W. Rosenhau, Z. Phys. 218, 437 (1969).

[23] S. Grossman, Phys. Lett. A 28, 162 (1968).

[24] S. Grossman and V. Lehmann, Z. Phys. 218, 449 (1969).

[25] P. Ipsen and T. Dossing (2003), 10th Nordic Meeting on Nuclear Physics, poster presentation, and private communication.

[26] I. Bena and M. Droz, Int. J. Mod. Phys. B 19, 4269 (2005).

[27] T. D. Lee and C. N. Yang, Phys. Rev. 87, 410 (1952). 
[28] C. N. Yang and T. D. Lee, Phys. Rev. 97, 404 (1952).

[29] N. Auerbach, Nucl. Phys. 76, 321 (1966).

[30] A. Volya, B. A. Brown, and V. Zelevinsky, Phys. Lett. B 509, 37 (2001).

[31] J. Dukelsky, C. Esebbag, and P. Schuck, Phys. Rev. Lett. 87, 066403 (2001).

[32] S. C. Pang, Nucl. Phys. A128, 497 (1969).

[33] S. C. Pang, A. Klein, and R. M. Dreizler, Ann. Phys. 49, 477 (1968).

[34] K. T. Hecht, Phys. Rev. 139, B794 (1965).

[35] K. T. Hecht, Nucl. Phys A493, 29 (1989).

[36] S. C. Pang and K. T. Hecht, J. Math. Phys. 8, 1233 (1967).

[37] G. G. Dussel, E. E. Maqueda, R. P. J. Perazzo, and J. A. Evans, Nucl. Phys. A460, 164 (1986).

[38] J. A. Evans, G. G. Dussel, E. E. Maqueda, and R. P. J. Perazzo, Nucl. Phys. A366, 77 (1981).

[39] J. Engel, K. Langanke, and P. Vogel, Phys. Lett. B 429, 215 (1998).

[40] Ginocchio, Nucl. Phys. 74, 321 (1965).

[41] R. W. Richardson and N. Sherman, Nucl. Phys. 52, 221 (1964).

[42] R. W. Richardson, J. Math. Phys. 6, 1034 (1965).

[43] J. Dukelsky and G. Ortiz, Int. J. Mod. Phys. E 15, 324 (2006).

[44] J. Dukelsky, C. Esebbag, and S. Pittel, Phys. Rev. Lett. 88, 062501 (2002).

[45] F. Pan and J. P. Draayer, Ann. Phys. 271, 120 (1999).

[46] F. Pan and J. P. Draayer, Phys. Rev. C 66, 44314 (2002).

[47] S. T. Belyaev, K. Dan. Vidensk. Selsk. Mat. Fys. Medd. 11, 31 (1959).

[48] V. Zelevinsky and A. Volya, Nucl. Phys. A 752, 325c (2005).

[49] V. Zelevinsky, B. A. Brown, N. Frazier, and M. Horoi, Phys. Rep. 276, 85 (1996).

[50] H. C. Pradhan, Y. Nogami, and J. Law, Nucl. Phys. A201, 357 (1973).

[51] O. Burglin and N. Rowley, Nucl. Phys. A602, 21 (1996).

[52] J. Dukelsky, G. G. Dussel, J. G. Hirsch, and P. Schuck, Nucl. Phys. A714, 63 (2003).

[53] R. A. Broglia, J. Terasaki, and N. Giovanardi, Phys. Rep. 335, 1 (2000).

[54] A. Volya, V. Zelevinsky, and B. A. Brown, Phys. Rev. C 65, 054312 (2002).

[55] K. Blum, Density matrix: theory and applications (Plenum Press, New York, 1996).

[56] L. D. Landau and E. M. Lifshitz, Statistical Physics (Pergamon Press, New York, 1978).
[57] V. V. Sokolov, B. A. Brown, and V. Zelevinsky, Phys. Rev. E 58, 56 (1998).

[58] C. Stoyanov and V. Zelevinsky, Phys. Rev. C 70, 014302 (2004).

[59] A. Volya and V. Zelevinsky, Phys. Lett. B574, 27 (2003), nucl-th/0307028.

[60] R.K.Pathria, Statistical Mechanics (ButterworthHeinemann, Oxford, 1996), 2nd ed.

[61] M. Guttormsen, M. Hjorth-Jensen, E. Melby, J. Rekstad, A. Schiller, and S. Siem, Phys. Rev. C 64, 034319 (2001).

[62] A. Schiller, M. Guttormsen, M. Hjorth-Jensen, J. Rekstad, and S. Siem, Phys. Rev. C 66, 024322 (2002), nuclth/0204045.

[63] W. H. Press, B. P. Flannery, S. A. Teukolsky, and W. T. Vetterling, Numerical recipes in $C$ : the art of scientific computing (Cambridge University Press, New York, 1992).

[64] S. Pratt, Phys. Rev. Lett. 84, 4255 (2000).

[65] M. Gaudin, Nucl. Phys. 20, 513 (1960).

[66] J. S. Langer, Phys. Rev. 134, A553 (1964).

[67] K. Huang, Statistical mechanics (Wiley, New York, 1987).

[68] M. S. Green, Phys. Rev. Lett. 1, 409 (1958).

[69] S. Koh, Phys. Lett. A 229, 59 (1997).

[70] R. Balian and J. S. Langer, Phys. Rev. 132, 958 (1963).

[71] M. Guttormsen, A. Bjerve, M. Hjorth-Jensen, E. Melby, J. Rekstad, A. Schiller, S. Siem,and A. Belic Phys. Rev. C 62, 024306 (2000).

[72] M. Guttormsen, R. Chankova, M. Hjorth-Jensen, J. Rekstad, S. Siem, A. Schiller, and D. J. Dean, Phys. Rev. C 68, 034311 (2003).

[73] V. Zelevinsky, Ann. Rev. Nucl. Part. Sc. 47, 237 (1996).

[74] A. Volya, B. A. Brown, and V. Zelevinsky, Prob. Theor. Phys. Supp. 146, 636 (2002).

[75] V. V. Flambaum and F. M. Izrailev, Phys. Rev. E 55, R13 (1997).

[76] V. V. Flambaum and F. M. Izrailev, Phys. Rev. E 56, 5144 (1997).

[77] M. Horoi and V. Zelevinsky, Phys. Rev. C 75, 054303 (2007).

[78] K. Maki, Phys. Rev. 148, 362 (1966).

[79] A. Bianchi, R. Movshovich, N. Oeschler, P. Gegenwart, F. Steglich, J. D. Thompson, P. G. Pagliuso, and J. L. Sarrao, Phys. Rev. Lett. 89, 137002 (2002).

[80] Y. Alhassid, L. Fang, and S. Schmidt (2007), condmat/0702304. 